\title{
Genome-Wide Identification and Characterization of the PERK Gene Family in Gossypium hirsutum Reveals Gene Duplication and Functional Divergence
}

\author{
Ghulam Qanmber ${ }^{1}{ }^{\circledR}$, Ji Liu ${ }^{1}{ }^{\circledR}$, Daoqian Yu ${ }^{1}$, Zhao Liu ${ }^{1}$, Lili Lu ${ }^{1}$, Huijuan Mo ${ }^{1}$, Shuya Ma ${ }^{1}$, \\ Zhi Wang ${ }^{1,2, *}$ and Zuoren Yang ${ }^{1,2, *}$ \\ 1 State Key Laboratory of Cotton Biology, Key Laboratory of Biological and Genetic Breeding of Cotton, \\ Institute of Cotton Research, Chinese Academy of Agricultural Sciences, Anyang 455000, China; \\ gqkhan12@gmail.com (G.Q.); liuji@caas.cn (J.L.); yudaoqian88@163.com (D.Y.); liuzhaocaas@163.com (Z.L.); \\ bioll1@126.com (L.L.); mohuijuan86@163.com (H.M.); msymsy89@126.com (S.M.) \\ 2 Zhengzhou Reseach Base, State Key Laboratory of Cotton Biology, Zhengzhou University, \\ Zhengzhou 450000, China \\ * Correspondence: wangzhi.12@163.com (Z.W.); yangzuoren4012@163.com (Z.Y.)
}

Received: 15 January 2019; Accepted: 1 April 2019; Published: 9 April 2019

\begin{abstract}
Proline-rich extensin-like receptor kinases (PERKs) are an important class of receptor kinases in plants. Receptor kinases comprise large gene families in many plant species, including the 15 PERK genes in Arabidopsis. At present, there is no comprehensive published study of PERK genes in G. hirsutum. Our study identified 33 PERK genes in G. hirsutum. Phylogenetic analysis of conserved PERK protein sequences from 15 plant species grouped them into four well defined clades. The GhPERK gene family is an evolutionarily advanced gene family that lost its introns over time. Several cis-elements were identified in the promoter regions of the GhPERK genes that are important in regulating growth, development, light responses and the response to several stresses. In addition, we found evidence for gene loss or addition through segmental or whole genome duplication in cotton. Gene duplication and synteny analysis identified 149 orthologous/paralogous gene pairs. $\mathrm{Ka} / \mathrm{Ks}$ values show that most GhPERK genes experienced strong purifying selection during the rapid evolution of the gene family. GhPERK genes showed high expression levels in leaves and during ovule development. Furthermore, the expression of GhPERK genes can be regulated by abiotic stresses and phytohormone treatments. Additionally, PERK genes could be involved in several molecular, biological and physiological processes that might be the result of functional divergence.
\end{abstract}

Keywords: G. hirsutum; GhPERK; sequence logos; phylogenetic analysis; cis-elements; gene duplication; abiotic stress; phytohormone stress

\section{Introduction}

Receptor kinases comprise large gene families in many plant species, including rice and Arabidopsis. The Arabidopsis receptor kinase family contains approximately 600 members and their homologs have been investigated in 20 different species [1-3]. The biological functions of most of these predicted receptor kinase proteins are unknown. However, receptor kinases are known to play essential roles in signal transduction, the response to external challenges in an ever-changing environment, hormone response pathways, plant growth and development, self-incompatibility, cell differentiation and symbiosis and pathogen recognition. Some studies have identified ligands that activate these receptor kinases and other signaling components as well $[4,5]$. Receptor kinases bind to a diverse array of molecules including steroids, polypeptides, carbohydrates and cell wall components, depending on their extracellular domain. Receptor kinases transduce signals across the plasma membrane via 
different signaling complexes found in eukaryotic cells other than plants and likely developed early during the evolution of multicellular organisms [2,5].

Previously, receptor kinases were classified into many distinct classes on the basis of motifs in their extracellular domains $[5,6]$. For example, the leucine-rich repeat (LRR) receptor kinase family is the largest class of Arabidopsis receptor kinases, including BRI1 and BAK1, which are involved in brassinosteroid (BR) perception [7-9]. Among the different classes of receptor kinases, gene duplications leading toward functional redundancy have also been observed [10]; for example, the CLV1 and ERECTA receptor kinases are evidence that functional redundancy exists [11-13]. Proline-rich extensin-like receptor kinases (PERKs) are an important class of receptor kinases. The Arabidopsis PERK family is similar to Brassica napus PERK1 and shares maximum sequence identity. In the Arabidopsis genome, a total of 15 PERK genes have been identified; however, their biological functions are largely unknown $[14,15]$. PERK1 is localized to the plasma membrane as predicted for a receptor kinase and the gene is induced rapidly in response to wounding in Arabidopsis [14]. Furthermore, PERK4 is a novel regulator of $\mathrm{Ca}^{2+}$ signaling that also mediates the early events leading up to the abscisic acid response in root tip growth $[16,17]$.

Plants face continuously changing environmental conditions during growth and development. Among these environmental challenges, abiotic and hormonal stresses are the crucial factors that affect plant growth and biological yield. Cotton is one of the leading fiber crops cultivated worldwide [18]. Various abiotic stresses and hormonal homeostasis, such as BR, play essential roles in its development and the quality of the cotton fiber $[19,20]$. Also, advancements in cotton genome sequencing [21-26] have made it possible to conduct a comprehensive investigation of cotton genes. In our study, we performed a systematic analysis of 207 PERK genes in 15 different plant species, including 33 PERK genes in G. hirsutum. Next, sequence logos, phylogenetic analysis, biophysical properties, exon/intron and protein motif distribution, promoter cis-element analysis, chromosomal distribution, gene duplication, synteny analysis and $\mathrm{Ka} / \mathrm{Ks}$ values were also determined. Furthermore, tissue-specific expression patterns, abiotic stress responses and functional roles with respect to BR signaling and other phytohormones were investigated for the G. hirsutum PERK genes. The present study will enable a detailed understanding of the molecular and biological functions of PERK genes in cotton.

\section{Results}

\subsection{Identification of PERKs}

In this study, we used various in silico approaches to identify a total of 207 PERK gene family members in 15 different plant species following confirmation with different tools such as PROSITE (http:/ / prosite.expasy.org/), SMART (http:/ / smart.embl-heidelberg.de/) and InterProscan 63.0 (http: //www.ebi.ac.uk/interpro/) (Table S1). The PERK gene family includes 15 genes from Arabidopsis, 15 from G. arboreum, 33 from G. hirsutum, 16 from G. raimondii, 16 from G. max, 8 from O. sativa, 14 from P. trichocarpa, 15 from S. bicolor, 9 from T. cacao, 23 from Z. mays, 12 from A. comosus, 10 from C. reinhardtii, 5 from P. patens, 10 from $S$. moellendorffii and 6 genes from P. taeda. We found that almost all selected plants have at least five PERK genes with G. hirsutum having the highest number (33) of PERK genes and P. patens having only five genes, indicating that PERK genes were subjected to a large scale expansion in higher plants. Because our main interest is in upland cotton, G. hirsutum, we compared the retrieved sequences of two different sequenced genomes of upland cotton including $\mathrm{BJI}$ and NAU and found no differences among the candidate genes. For the following analyses we used the gene sequences retrieved from the NAU genome sequence database. Here, allotetraploid upland cotton had more than double the number of PERK genes compared to diploids cotton species such as G. arboreum and G. raimondii, illustrating the effect of polyploidy on the PERK gene family in G. hirsutum which is derived from the hybridization of two progenitor species resembling the diploids G. arboreum and G. raimondii. 
We next determined the biophysical properties of the GhPERK family members including locus ID, the corresponding chromosome, start and end points, strand polarity, gene length (bp), CDS length (bp), protein length (aa), predicted protein molecular weights (MW) and isoelectric points (pl), predicted masses and predicted cellular localization. We found that 14 GhPERK genes originated from the At sub-genome, 15 from the Dt sub-genome and four were from scaffolds of the G. hirsutum genome. Predicted protein lengths range from 178-884 aa, with molecular weights (MW) of 19,755.66-93,594.32 Da for GhPERK7 and GhPERK14, respectively. Moreover, the cellular localizations of all GhPERK proteins were related to the plasma membrane. All other estimated biophysical properties are shown in Table S2.

\subsection{Sequence Logos and Phylogenetic Analysis}

To check whether the PERK family proteins were conserved during evolution, we generated the sequence logos of the conserved amino acid residues in Arabidopsis, rice and upland cotton G. hirsutum (Figure 1). We found that the sequence logos among the three species were highly conserved across the $\mathrm{N}$ and $\mathrm{C}$ termini such as D [4], F [5], H [22], T [25], G [27], T [26], G [28], A [29], P [30], E [31], G [32], D [33], V [34], G [35], L [36], E [37], L [38] and so forth. Also, no compositional bias of any specific pattern of conserved amino acid residues was observed in sequence logos of Arabidopsis, rice and G. hirsutum. As sequence logos provide a more precise description of sequence similarity than consensus sequences, significant features of the alignment, patterns in sequence conservation and assist to discover and analyze those patterns [39]. In this regard, PERK protein sequence logos in Arabidopsis, rice and G. hirsutum will assist to discover, analyze and evaluate the pattern of PERK protein sequence conservation in other plant species.

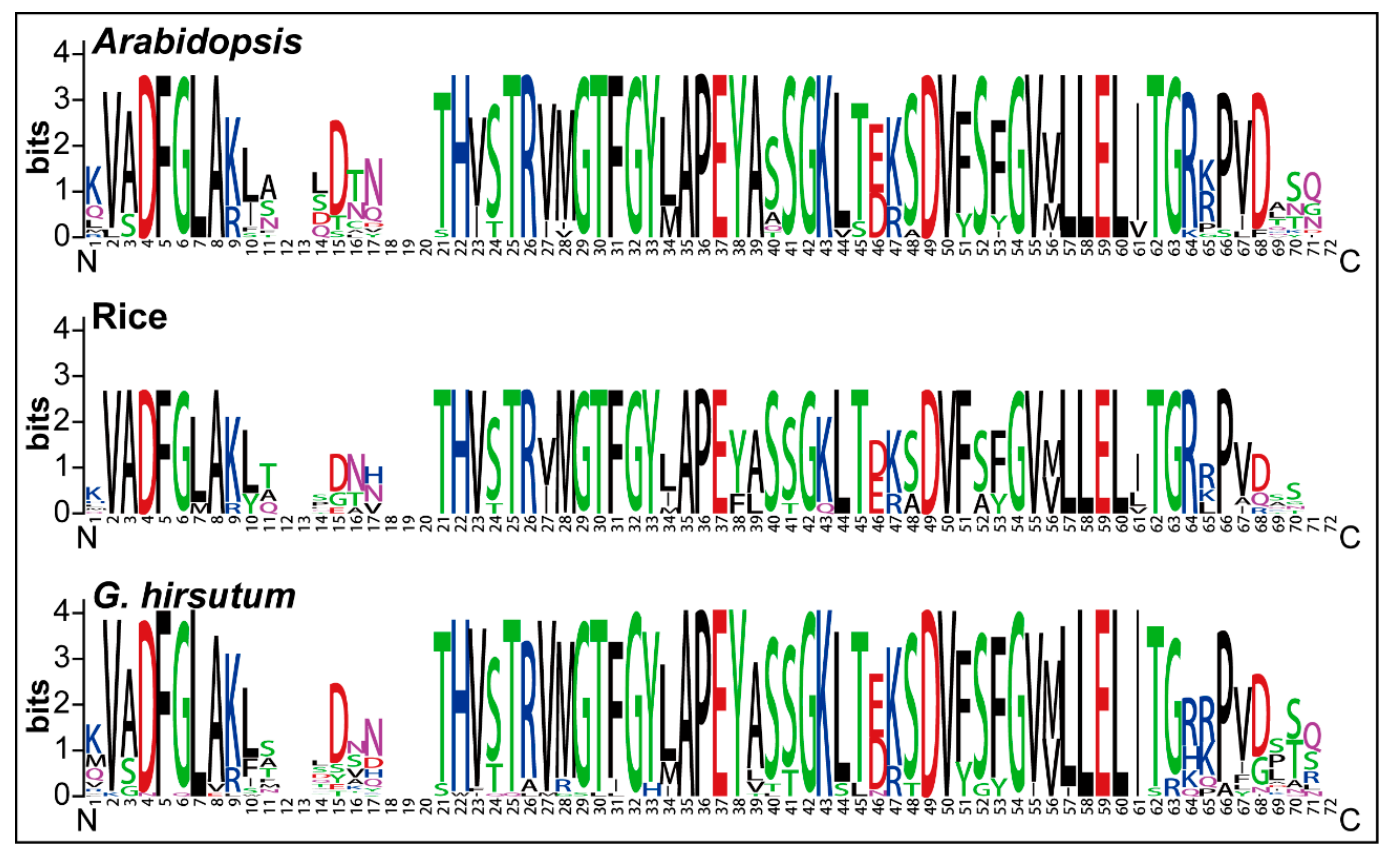

Figure 1. Sequence logos of conserved amino acid residues in Arabidopsis, Rice and G. hirsutum.

The phylostratum analysis of PERK gene family identified the earliest plant lineage as PERK genes were present in C. reinhardtii (chlorophyte), an earliest plant lineage (Figure 2A). Further, the PERK genes were present in A. comosus (angiosperm), P. taeda (gymnosperm), P. patens (bryophytes), S. moellendorffii (lycophytes), dicots (A. thaliana, G. arboreum, G. hirsutum, G. raimondii, G. max, P. trichocarpa and T. cacao) and monocots (O. sativa, S. bicolor and Z. mays). These results indicated that PERK genes were originated from early land plants phylostratum and potential orthologous genes of PERK are present throughout pant kingdom. Next to construct the evolutionary relationships among 
the 207 PERK genes from 15 different plant species including A. thaliana, G. arboreum, G. hirsutum, G. raimondii, G. max, P. trichocarpa, T. cacao, O. sativa, S. bicolor, Z. mays, A. comosus, C. reinhardtii, P. patens, S. moellendorffii and P. taeda, a Maximum likelihood (ML) tree was generated. Phylogenetic analysis grouped all PERK proteins into four clades (PERKa-d) on the basis of their sequence homologies (Figure 2B).

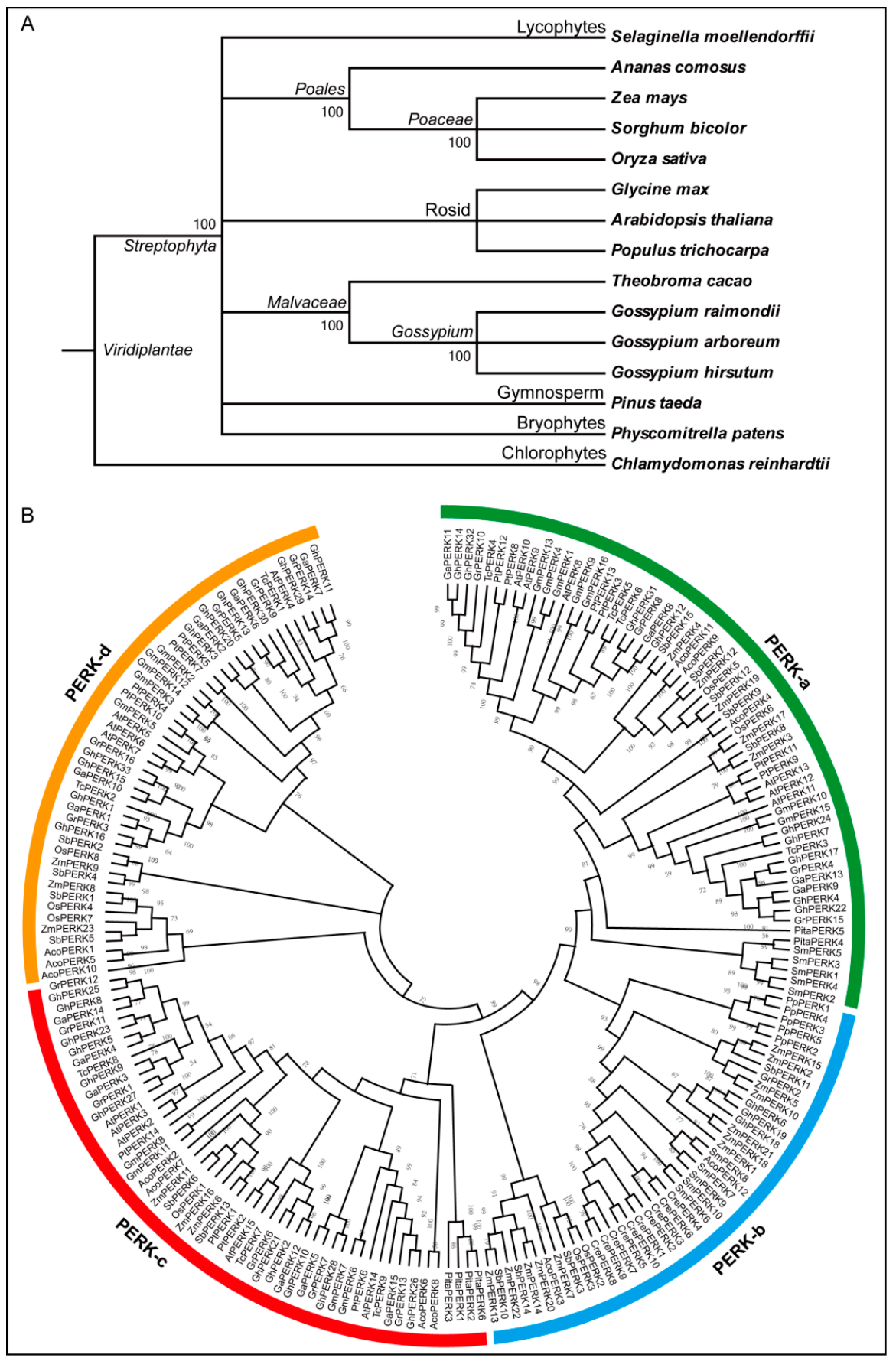

Figure 2. Phylogenetic analysis of PERK genes from 15 plant species. (A) The phylostratum analysis of PERK gene family. (B) Phylogenetic tree divided all 207 PERK genes into four groups from PERK-a to PERK-d. Prefixes such as At, Ga, Gh, Gr, Gm, Pt, Tc, Os, Zm, Sb, Aco, Cre, Pp, Sm and Pita were used before the names of A. thaliana, G. arboreum, G. hirsutum G. raimondii, G. max, P. trichocarpa, T. cacao, O. sativa, Z. mays, S. bicolor, A. comosus, C. reinhardtii, P. patens, S. moellendorffii and P. taeda PERK genes respectively. Bootstrap values were also mentioned near node of each branch. 
In the phylogenetic analysis, the PERK-a clade contained 62 members, PERK-b contained 44 members, PERK-c contained 53 members and PERK-d contained 48 members of the PERK protein family. PERK-a clade contains genes from S. moellendorffii (lycophytes), P. taeda (gymnosperm), A. comosus (angiosperm), dicots and monocots except C. reinhardtii (chlorophyte) and P. patens (bryophytes), indicated the evolution of these PERK genes after the split of lycophytes, chlorophyte and bryophytes. While PERK-b clade contains PERK genes from all plant species except P. taeda. Similarly, PERK-c clade lacks the PERK family members from S. moellendorffii, C. reinhardtii and P. patens. While PERK-d clade lacks the PERK genes from S. moellendorffii, C. reinhardtii, P. patens and $P$. taeda. Surprisingly, PERK genes from C. reinhardtii were only present in clade PERK-b. Interestingly, $P E R K$ genes from dicot and monocot plant species were randomly distributed to all four clades. Moreover, PERK genes from G. hirsutum were present in almost all clades with PERK-c having highest number (11) of GhPERK genes and PERK-b having only three GhPERK genes. These results demonstrated that PERK-b clade is an ancient group of PERK genes having PERK gene family members from almost all plant species except $P$. taeda. Further, the phylogenetic analysis indicated that G. hirsutum experienced significant gene family expansion, because it has almost double the number of PERK genes compared to the other plant species except Z. mays. At least eight pairs or triplets of GhPERK proteins cluster together in the phylogenetic tree, which can be interpreted as evidence of gene duplication. All species except for G. raimondii and S. bicolor had gene pairs derived from the same node, demonstrating that the PERK genes in all species included in our study have experienced gene duplication events that resulted in PERK gene family expansion. However, gene duplication among the different groups varies for the different species. These results indicate that gene duplication is the main contributor toward PERK gene family expansion in all plant species. These results could be considered as evidence for lineage-specific expansion of the PERK genes after the divergence of dicots and monocots. Further, a NJ tree was constructed for the cotton PERK proteins in order to investigate the relationship between the common ancestors of diploid (G. arboreum and G. raimondii) and allotetraploid cottons (G. hirsutum) (Figure S1). The tree grouped all cotton PERK proteins into six clearly-defined clades, each of which contains proteins from both diploid and allotetraploid cotton species. The clusters provide evidence confirming that G. hirsutum is the result of hybridization between the two diploid cotton species G. arboreum and G. raimondii.

\subsection{Exon/Intron, Protein Motif and cis-element Analysis}

It has been previously reported that the exon/intron distribution pattern in a gene is related to its biological function. Exon/intron analysis along with phylogenetic tree results showed that all GhPERK genes lack introns and that similar genes were clustered close to one another in the same groups (Figure S2A). Furthermore, the protein motif distribution patterns of the GhPERK proteins revealed that similar motifs had conserved distribution patterns, even in the case of GhPERK19, GhPERK18 and GhPERK6 that lacked certain motifs (Figure S2B). In fact, we found that all GhPERK genes showed conserved patterns of gene structure (exon/intron) and protein motif distribution for the At and Dt sub-genomes even though gene duplication occurred long ago during evolution.

Because gene expression is regulated by promoters and by the binding of transcription factors to cis-elements located in upstream regions, cis-element analysis is important to understanding gene regulation and function [27,40]. We identified cis-elements in the promoters of the GhPERK genes and categorized them based on their functional relevance (Table S3). The identified cis-elements were classified with respect to their functions in growth and development and light and stress responses. All of the GhPERK genes had various cis-elements for different responses such as the GCN4 and Skn-1 motifs (endosperm expression), the as-2-box (shoot-specific expression), the 3-AF1 binding site, AC-II, the CCGTCC-box, Sp1 and circadian for growth and development, ACE, AE-box, ATCT-motif, Box 4, Box I, Box II, G-box, GA-motif, GAG-motif, GATA-motif, GC-motif, GT1-motif, I-box, L-box, Sp1, TCT-motif and chs-CMA1a for light response and MBS and TC-rich repeats (For stress and defense), LTR (for low temperature), Box-W1 (fungal elicitor), ARE, CGTCA-motif, GARE-motif, 
HSE, TCA-element, TCCACCT-motif, TGACG-motif, TGA-element and TCCC-motif for different stress responses. Additional cis-elements that regulate responses to different hormones such as auxin (TGA-element and AuxRE-core), salicylic acid (TCA-element and SARE), gibberellin (TATC-box, P-box and GARE-motif), MeJA (CGTCA-motif and TGACG-motif) and ethylene (ERE-box) were also identified in the promoter regions of many GhPERK genes.

\subsection{Chromosomal Distribution, Gene Duplication and Synteny Analysis}

All identified GhPERK genes were mapped to their corresponding chromosomal loci. The results showed that 14 genes mapped to At sub-genome chromosomes, 15 to Dt sub-genome chromosomes and four to scaffolds (Figure S3). Most of the chromosomes contained only a single GhPERK gene, although D01 contained three genes and there were no GhPERK genes identified on chromosomes D02, A03, A09 and its homolog D09. The absence of any PERK genes on A03 is supported by a previous study in which a translocation between chromosomes A02 and A03 was reported. Furthermore, the absence and uneven distribution of GhPERK genes on some At and Dt sub-genome chromosomes and scaffolds indicates that gene loss may have occurred during evolution but an incomplete genome assembly could also be a factor.

To study the locus relationships among the GhPERK genes, we identified the orthologous/ paralogous gene pairs for the At and Dt sub-genomes of upland cotton (G. hirsutum). Upland cotton is an allotetraploid crop that is a model species for the study of natural polyploidy [26]. Synteny analysis indicated that several gene loci are highly conserved between the At and Dt sub-genomes of upland cotton (Figure 3). As described above, upland cotton resulted from the hybridization between the ancestors of the diploid species G. arboreum and G. raimondii [28,41]. Similar to previous findings, our study also demonstrated that the At as well as the Dt sub-genomes have orthologous in the $\mathrm{A}$ (G. arboreum) or D (G. raimondii) genomes and the observation that the genes from any one from these four genomes (At, Dt, A and D) have the corresponding orthologous from the other three genomes provides evidence that cotton PERK genes may not have experienced genomic arrangements during polyploidization. A total of 149 orthologous/paralogous gene pairs were identified, of which 10 pairs were attributed to segmental duplication to form paralogous gene pairs within the At or Dt sub-genomes (Table S4). From these results we assumed that paralogous gene pairs were mainly contributed by segmental or whole genome duplication prior to polyploidization. We also identified these duplicated gene pairs in diploid cottons and their presence demonstrated that gene duplication occurred before the hybridization and formation of the allotetraploid species G. hirsutum (Figure 3 and Table S4).

During evolution, duplicated gene pairs can experience functional divergence resulting in neo-functionalization, sub-functionalization, or non-functionalization [42]. To determine the nature and extent of selection pressure on these duplicated gene pairs, we calculated their $\mathrm{Ka} / \mathrm{Ks}$ values (Table S4). The Non-synonymous (Ka) and synonymous (Ks) divergence values showed that for 129 duplicated gene pairs, the Ka/Ks ratios were $<0.5$, while for another 17 pairs, Ka/Ks was between 0.5 and 1.0. The only exception was that the $\mathrm{Ka} / \mathrm{Ks}$ ratio for three gene pairs was $>1.0$, illustrating that cotton the PERK genes have undergone strong purifying selection, since Ka/Ks was $<1.0$ for 146 out of 149 duplicated gene pairs and only three duplicated gene pairs showed positive selection pressure. 


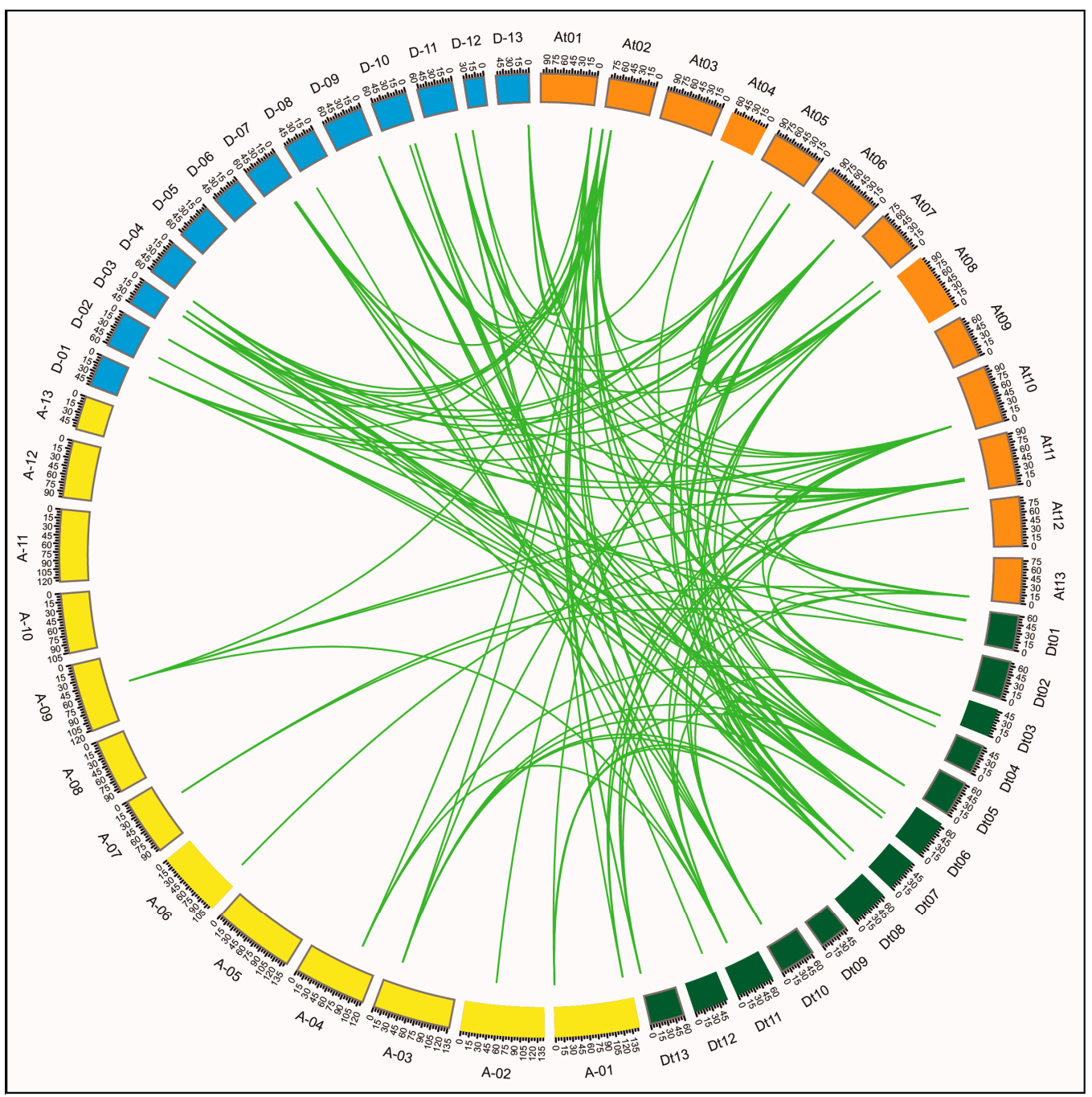

Figure 3. Gene duplication among cotton PERK genes including G. hirsutum (At and Dt sub-genome), G. arboreum (A-genome) and G. raimondii (D-genome). Green lines exhibited orthologous/paralogous pairs. At01-At13 (orange blocks) indicated chromosomes of At sub-genome and Dt01-Dt13 (dark green blocks) represented chromosomes of Dt sub-genome. Similarly, A-01 to A-13 (yellow blocks) and D-01 to D-13 (light blue blocks) represented G. arboreum and G. raimondii chromosomes, respectively.

\subsection{Tissue-Specific Gene Expression Patterns and Abiotic Stress Responses}

Regulated gene expression is critical for normal plant growth and development. Expression patterns can provide clues into the biological functions of genes, so we examined the expression patterns of the GhPERK genes in different cotton tissues. First, we analyzed GhPERK gene expression in published transcriptomic data downloaded from NCBI (https:/ / www.ncbi.nlm.nih.gov/pmc/ articles/PMC4482290/) for 22 different tissues including roots, stems and leaves, reproductive tissues such as torus, petals, stamens, pistils, calyces and ovules sampled at -3, -1, 0, 1, 3, 5, 10, 20, 25 and 35 days post-anthesis (DPA) and fibers sampled at 5, 10, 20 and 25 DPA. Heat mapping of gene expression indicated that genes with similar expression levels clustered close together and that the expression patterns for many of the genes differed considerably in the different tissues (Figure 4A). Furthermore, because the fiber is the product harvested from cotton, we evaluated the expression of GhPERK genes in previously published RNA-seq data from two lintless/fuzzless mutants named M1l and $M 2 l$ [43] and generated a heat map of the expression levels (Figure 4B). The map showed that expression of one gene (GhPERK1) was up-regulated and two genes (GhPERK13 and GhPERK26) were down-regulated in the M1l mutant, while the three genes display the reverse expression patterns in the 
M2l mutant. However, the relative expression of 10 genes was the same in both mutants. Overall, 13 GhPERK genes were down- while seven GhPERK genes were up-regulated for both mutants, indicating both positive and negative roles in cotton fiber formation (Figure $4 \mathrm{~B}$ and Table S5).

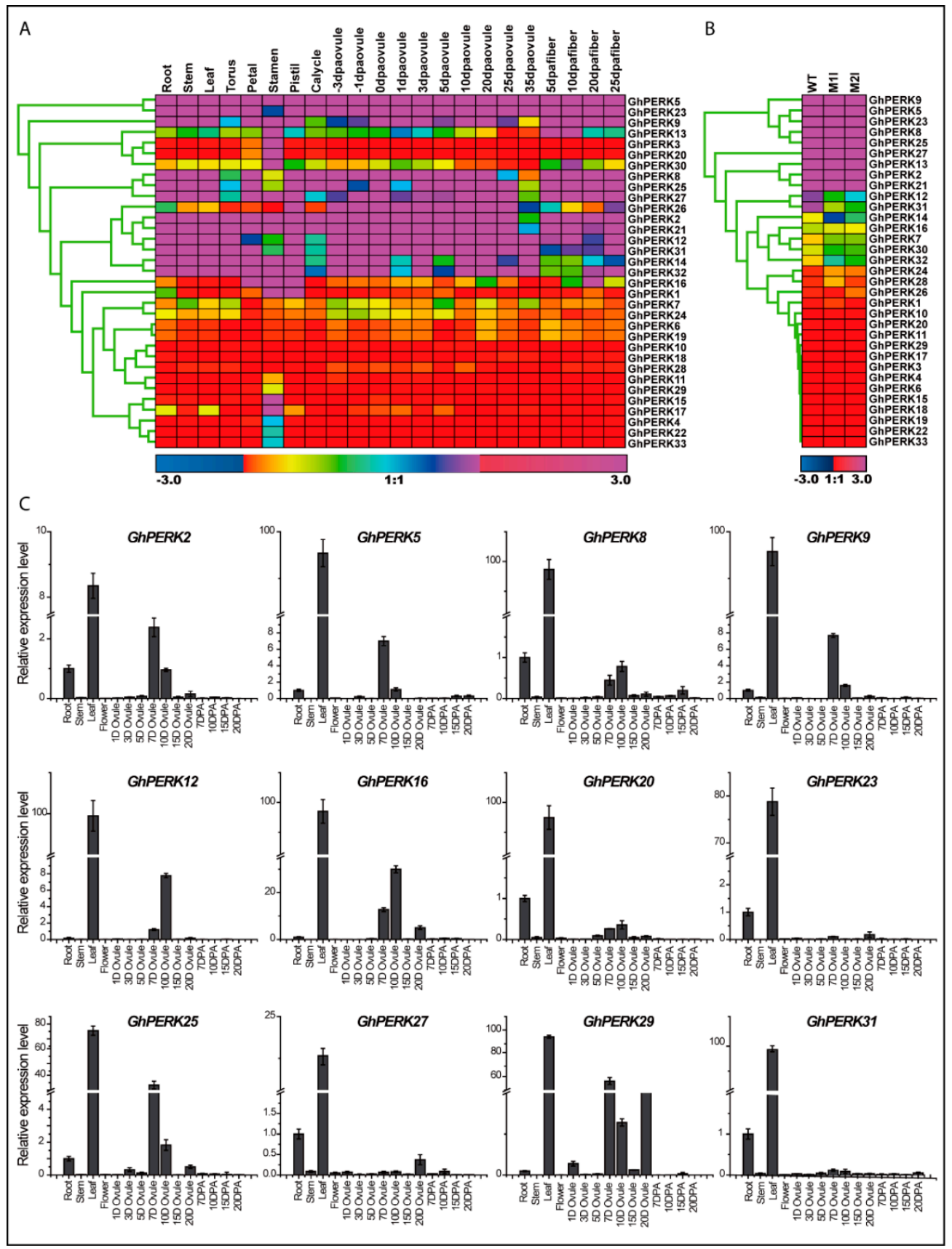

Figure 4. Relative expression level of GhPERK genes in different tissues of cotton plant. (A) Heat map of 33 GhPERK genes in 22 different tissues. Data was obtained from publicly available transcriptomic data and color bar (down) represented expression level. (B) Heat map of GhPERK genes relative expression in two fuzzless/lintless mutants (M1l and M2l) in comparison to WT plants. Data was obtained from published RNA-seq data and color bar (down) indicated the value of expression level. (C) Relative expression level by qRT-PCR analysis of selected GhPERK genes. Error bars indicated the standard deviations (SD) among three independent biological repeats.

From here, we selected 12 of the most down-regulated genes and examined their tissue-specific expression patterns by qRT-PCR analysis in roots, stems, leaves, flowers, ovules $(1,3,5,7,10,15$ and 
$20 \mathrm{DPA})$ and fibers $(7,10,15$ and $20 \mathrm{DPA})$. Interestingly, the results were generally similar to analyses of previously published transcriptomic and RNA-seq data, as all 12 genes were highly expressed in leaves and also during some stages of ovule development. However, all estimated GhPERK genes did not show any obvious contribution in fiber development and other tissues except for root by some genes (Figure 4C). Together with these results, we speculated that GhPERK genes played roles in leaf development as well as for ovule development with few exceptions.

Cotton plants face several abiotic stress challenges during their growth and development; therefore, we next evaluated GhPERK gene expression in response to various abiotic stresses. First, we analyzed the relative expression of GhPERK genes in publicly-available transcriptomic data for changes related to abiotic stresses including cold, heat, salt and PEG (drought) at several time points [24]. A heat map showed that the expression of all GhPERK genes was up- or down-regulated under different treatments at different time points (Figure 5A). Genes displaying similar responses clustered close together. In addition, we used qRT-PCR to estimate the expression levels of selected genes in response to cold, heat, $\mathrm{NaCl}$ and PEG after 1, 2, 4 and 6 hours of treatment (Figure 5B). Similar to the transcriptome analysis, most of the GhPERK genes were found to be down-regulated at different time points for most of the abiotic stress treatments. GhPERK12, GhPERK27 and GhPERK29 expression was mostly up-regulated at different time points for all of the tested stress treatments with a few exceptions. However, the expression of GhPERK8, GhPERK16, GhPERK20, GhPERK25 and GhPERK31 was down-regulated for most or all treatments. From these results, we can assume that expression of GhPERK genes shows sensitivity to abiotic stresses and most genes are generally down-regulated in response to the four different abiotic stresses.

\subsection{GhPERK Gene Expression in Response to Phytohormone Treatments}

To investigate the roles of GhPERK genes in response to the phytohormone brassinosteroid (BR), first we analyzed the tissue-specific expression levels of selected genes in the cotton brassinosteroid mutant Pag1 [20] compared to wild type cotton. Interestingly, the results of qRT-PCR analyses indicated that all tested genes were up-regulated and had high mRNA levels in all tissues examined including hypocotyls, stems and 10 and 20 DPA fibers compared to WT plants. The relative expression of all 12 of these genes was much higher in leaves of the WT compared to the mutant (Figure 6A). These results may help to explain why all GhPERK genes did not show high expression levels during fiber development and in the other tested tissues in the WT plants because Pag1 mutant plants display short stature with no obvious fiber development. The GhPERK genes could be responsible for this altered morphology through brassinosteroid catabolism but a more extensive investigation may be needed to explore the complex gene regulation. These results demonstrate the negative regulation of GhPERK genes in the brassinosteroid pathway. 


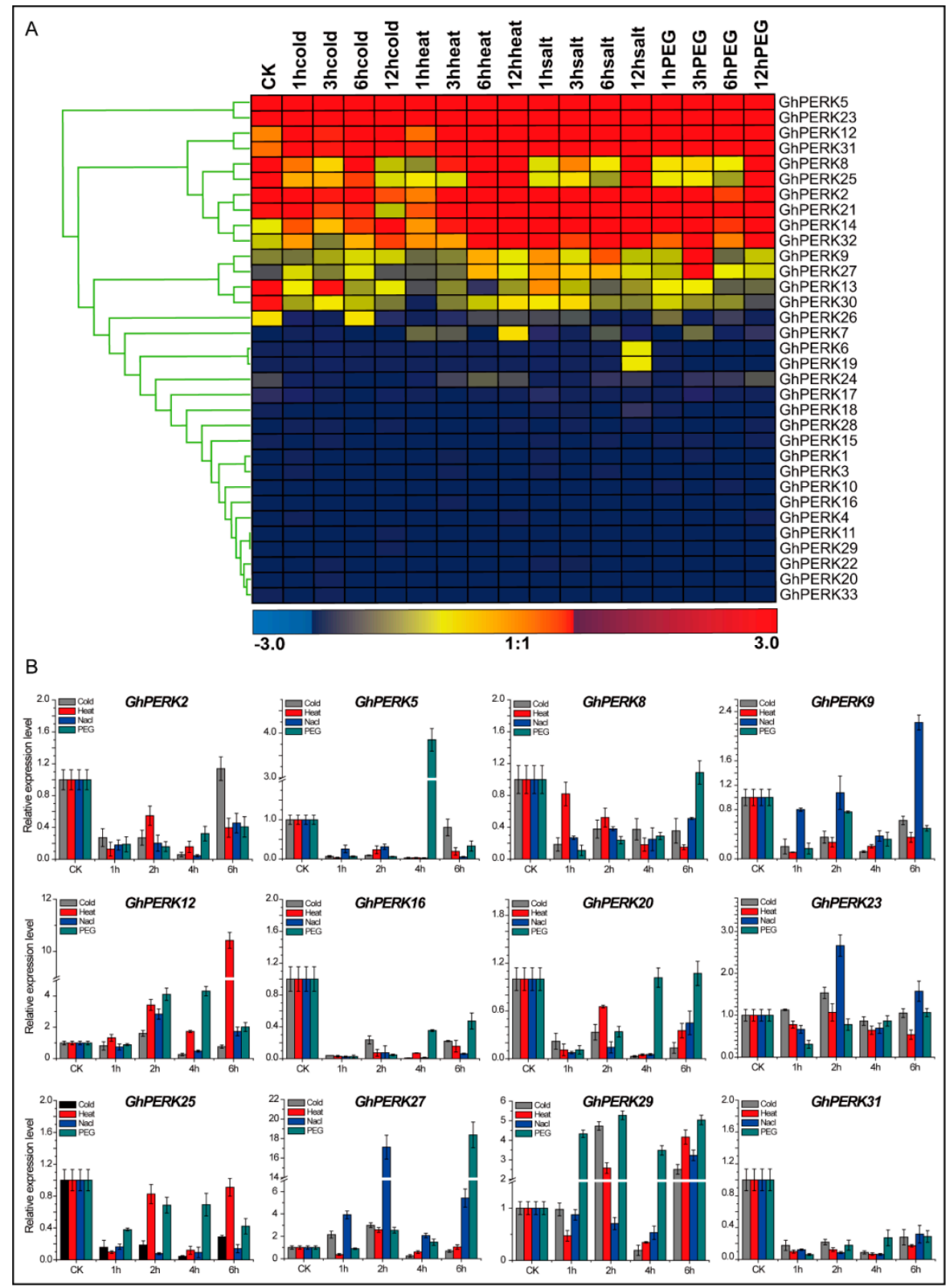

Figure 5. Expression analysis of GhPERK genes for four different abiotic stresses including cold, heat, $\mathrm{NaCl}$ and PEG. (A) Heat map of GhPERK genes constructed by publicly available transcriptomic data. Color bar (down) depicted the level of response for that gene. (B) qRT-PCR expression pattern of GhPERK genes under abiotic stresses. The error bars exhibits standard deviations (SD) among three independent biological repeats. 


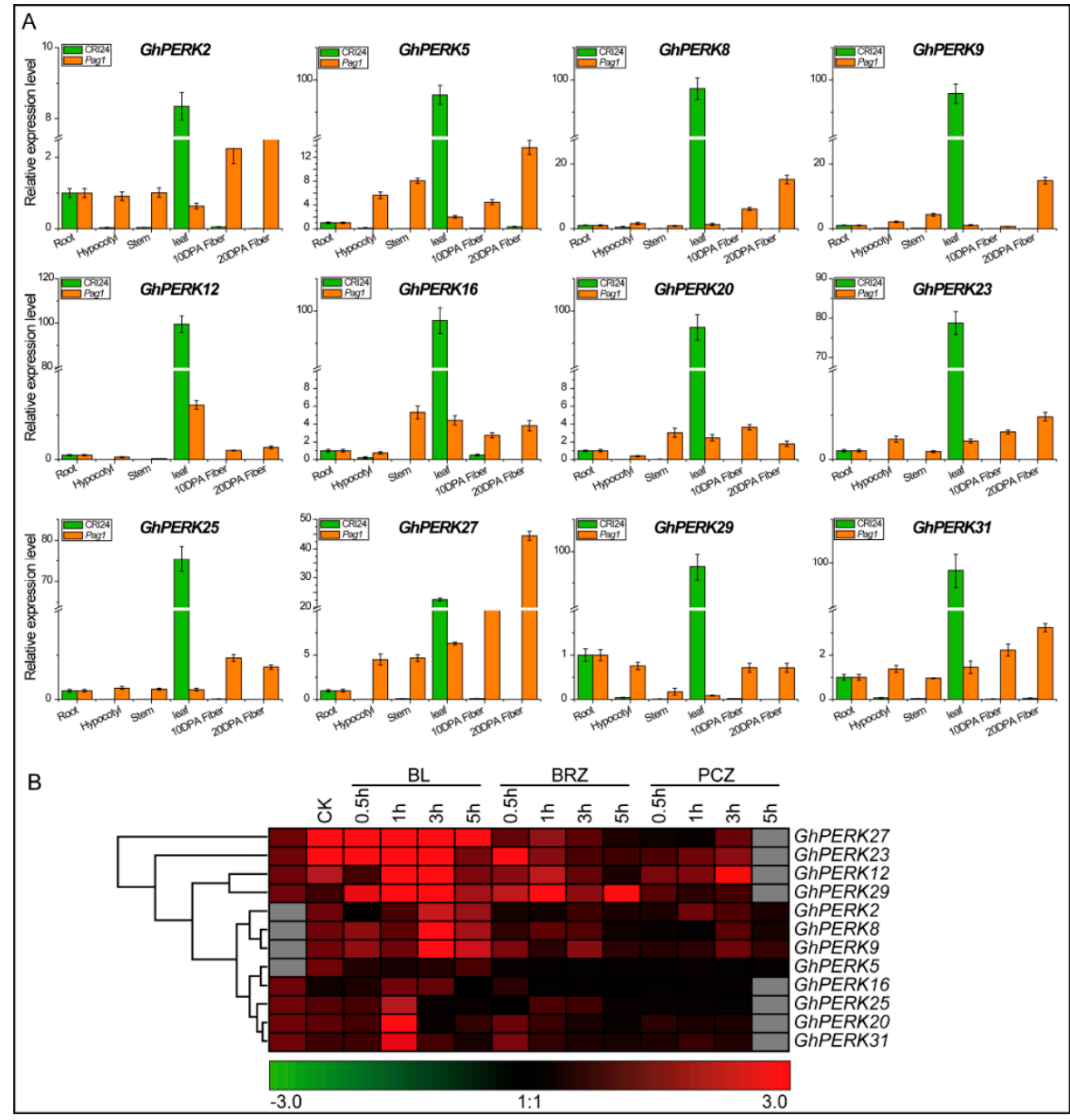

Figure 6. Expression pattern analysis of GhPERK genes for brassinosteroid (BR) hormone. (A) Relative expression level of GhPERK genes in Pag1 (cotton brassinosteroid mutant) and CRI24 (WT) evaluated by qRT-PCR analysis. (B). Heat map generated by the relative expression values estimated by qRT-PCR analysis on the exposure of BL, BRZ and PCZ treatment. Error bars exhibits standard deviations (SD) among three independent biological repeats.

In addition, to determine whether GhPERK gene expression can be regulated by brassinosteroids, we used qRT-PCR to examine the expression levels of GhPERK genes in cotton seedlings treated with the active form of brassinosteroid BL (brassinolide) and two brassinosteroid biosynthesis inhibitors, BRZ (brassinozole) and PCZ (propiconazole), for 0.5, 1, 3 and 5 hours (Figure 6B). Interestingly, all GhPERK genes were found to be regulated by BL, BRZ and PCZ treatments with no specific patterns. GhPERK27 and GhPERK23 expression was up-regulated by BL but was down-regulated by BRZ and PCZ at different time points in the experiment. However, GhPERK5 expression was down-regulated at all time points for all treatments. In addition, none of the genes showed patterns of expression that were indicative of complex regulation. Taking all the results together, we hypothesize that the GhPERK genes are regulated by BR and play roles in the BR signaling pathway.

It has been reported previously that the expression of Arabidopsis PERK4 responds to abscisic acid (ABA) during root tip growth $[16,17]$. In addition to the many phytohormone responsive cis-elements identified in this study, we evaluated the GhPERK genes for their responses to phytohormones including GA, IAA, SA and MeJA after 0.5, 1, 3 and 5 hours of treatment (Figure 7). Here, we also observed differential gene expression for the various genes in response to the different hormones 
over the 5-hour course of the experiment. GhPERK8, GhPERK9, GhPERK12, GhPERK23, GhPERK27 and GhPERK29 expression was mostly up regulated with a few exceptions. However, GhPERK5 was the one gene that was down-regulated when the plants were exposed to all of the hormones at all time points. Moreover, the GhPERK genes displayed no specific expression patterns for any of the hormones, suggesting that the GhPERK genes experienced strong functional divergence following gene duplication during evolution.

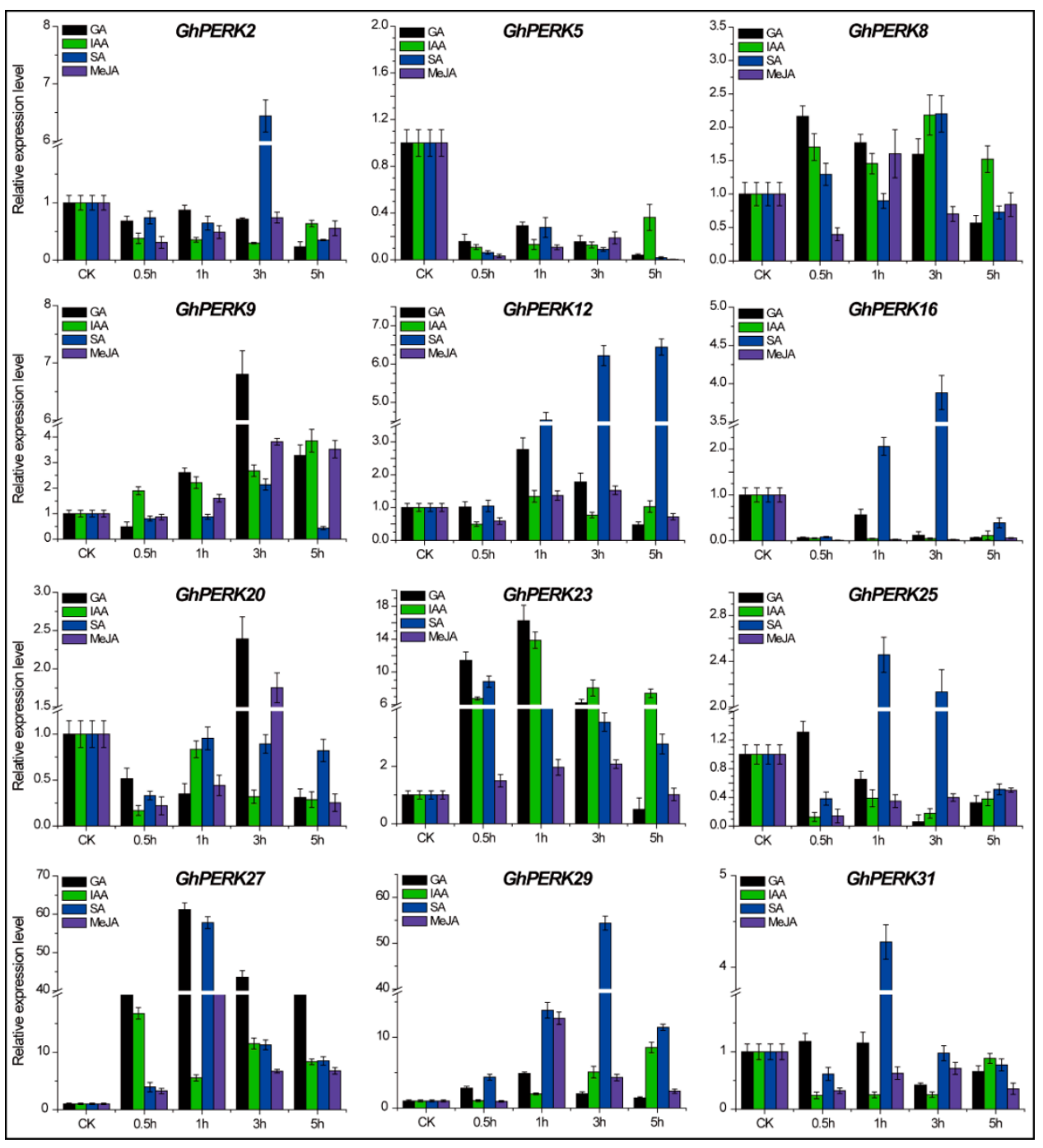

Figure 7. Expression pattern analysis of GhPERK genes under different phytohormonal stresses including GA, IAA, SA and MeJA in cotton seedlings by qRT-PCR analysis. Error bars indicated standard deviations (SD) among three independent biological experiments.

\subsection{Regulatory Sub-Networks Involving GhPERK and Other G. hirsutum Genes}

Co-expressed genes show similar increases or decreases in transcription levels in different samples [44]. The knowledge about neo- or sub-functionalization of gene paralogues needs to study gene modules along with molecular evolutionary approaches. Co-expression networks are conserved across species and even across distinct kingdoms of life clearly indicating cross-kingdom orthologs presence. Co-expression networks have emerged as an important tool to rapidly infer the functional relatedness among genes belonging to similar functions/pathways [29]. The PERKs are known to influence several developmental, signaling and physiological processes in plants. The co-expression between GhPERKs and other G. hirsutum genes were analyzed using cCNET and co-expression genes were identified. We found that two GhPERK genes were co-expressed with other G. hirsutum 
genes. GhPERK3 was co-expressed with 37 G. hirsutum genes (Figure 8A), while GhPERK20 had 30 co-expressed G. hirsutum genes (Figure 8B). Further, Gene Ontology (GO) showed that these genes were enriched in $17 \mathrm{GO}$ terms (FDR < 0.05) related to enzyme inhibitor activity, polygalacturonase activity, glycoprotein binding, calcium ion binding, actin binding, zinc ion binding and defense response (Table S6). Similarly, Kyoto Encyclopedia of Genes and Genomes (KEGG) enrichment analysis indicated that most of co-expressed genes were involved in plant-pathogen interaction, pentose and glucuronate interconversions, regulation of actin cytoskeleton, nitrogen metabolism, monoterpenoid biosynthesis and microbial metabolism in diverse environments (Table S6).

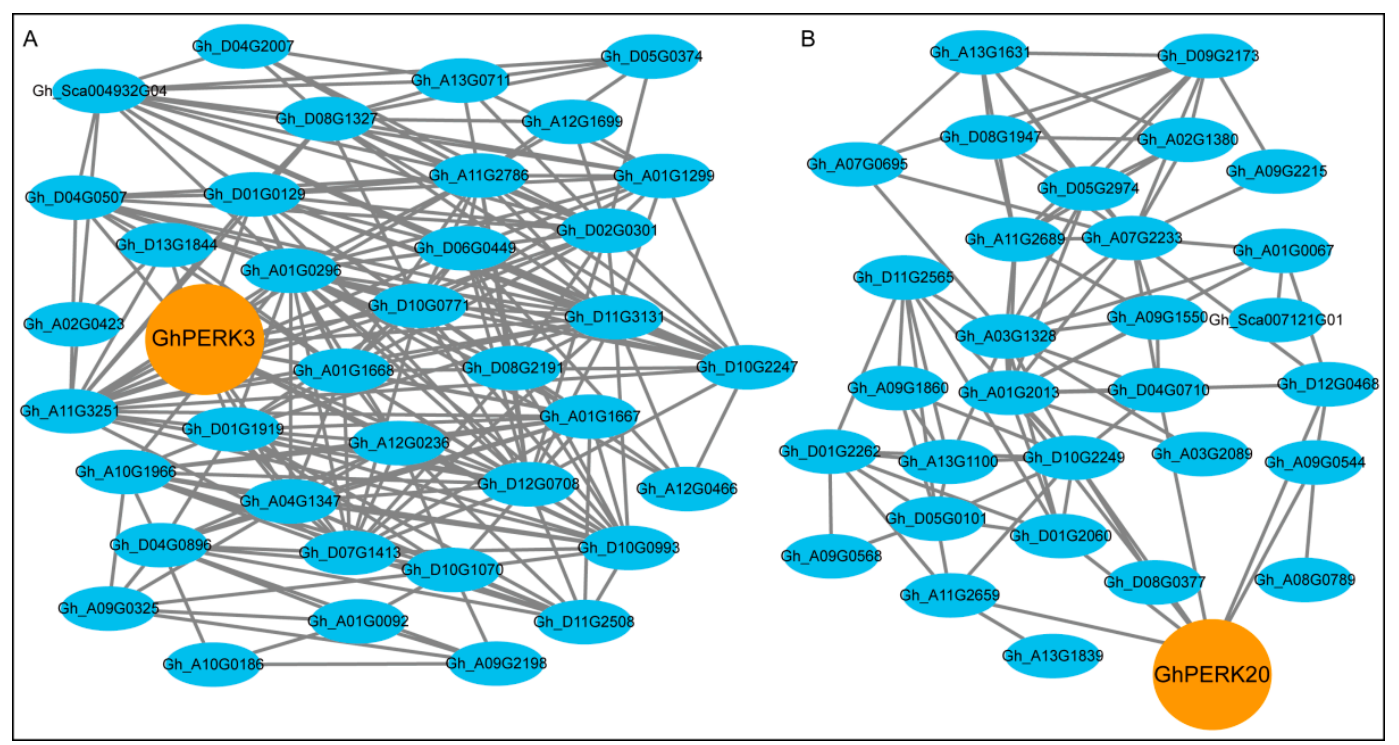

Figure 8. Co-expression analysis of GhPERK genes visualized by Cytoscape software. (A) Co-expression sub-network for GhPERK3 gene. (B) Co-expression sub-network for GhPERK20 gene. Nodes are connected by lines to show the interaction of that gene to other co-expressed gene.

\section{Discussion}

Several previous studies explored the molecular and physiological functions of PERK genes in various plant species $[1,14-17]$ but no studies describing the PERK genes and their functions in cotton have been published at present. Cotton is the largest and most important source of fiber for textile industry [30]. Ours the first ever study to identify the PERK genes in diploid and allotetraploid species of cotton and to analyze their evolutionary relationships, gene duplication and selection pressure and expression in response to various abiotic stresses and hormone treatments. Recent advances in cotton genome sequencing and genomics made it possible to conduct a comprehensive study of cotton PERK genes and investigate their potential functions. Our research identified and analyzed PERK genes in G. arboreum, G. hirsutum and G. raimondii but we mainly focused on the genes in G. hirsutum. The results of our study will provide basic information and serve as an important resource for further investigations into PERK gene function in cotton.

\subsection{GhPERK Genes Show Evolutionary Conservation}

In the present study, we classified PERK protein family members from S. moellendorffii (lycophytes), P. taeda (gymnosperm), A. comosus (angiosperm), C. reinhardtii (chlorophyte), P. patens (bryophytes), seven dicots (A. thaliana, G. arboreum, G. hirsutum, G. raimondii, G. max, P. trichocarpa and T. cacao) and three monocots (O. sativa, S. bicolor and Z. mays) into four phylogenetic clades. The phylogenetic analysis showed that the PERK proteins from S. moellendorffii, A. comosus and P. taeda divided the PERK proteins into these four clades. The phylostratum analysis of PERK gene family identified the earliest plant lineage as PERK genes were present in C. reinhardtii (chlorophyte), indicated that 
PERK genes were originated from early land plants phylostratum and potential orthologous genes of PERK are present throughout pant kingdom. Phylogenetic tree showed that PERK-b clade is an ancient group having PERK gene family members from all selected plant species except $P$. taeda. While, PERK-d clade could be more advance than others, lacking PERK genes from S. moellendorffii, P. taeda, C. reinhardtii and P. patens. The presence of PERK genes in each selected plant species, with 33 PERK genes from G. hirsutum and only five PERK genes from P. patens, demonstrated that PERK genes are evolutionary conserved and experienced a large scale expansion in higher plants. Moreover, cotton and cacao PERK genes depicted close relationship during phylogenetic analysis strengthening the previous finding that cotton and cacao share a common ancestor [25]. Sequence logos for conserved amino acid residues were highly conserved in Arabidopsis, rice and G. hirsutum at both the $\mathrm{N}$ and $\mathrm{C}$ termini and there was no compositional bias in any conserved amino acid residues indicating that the PERK genes are evolutionarily conserved which will help to identify the pattern of PERK protein sequence conservation in other plant species. The WOX and $Y A B B Y$ gene families were also found to be evolutionarily conserved and exhibit similar conserved sequence logos [31,45].

The promoter sequences of the GhPERK genes contain various predicted cis-elements that are specific for plant growth, development and light and stress responses. Transcription is regulated by the binding of transcription factors to upstream cis-acting regulatory elements. Many studies have shown the important role that light plays in the processes of plant growth and development [46]. A number of cis-elements such as the TCA and CGTCA elements for MeJA and SA stress, respectively [32,47], dehydration-response elements [48], abscisic acid (ABA) [49], heat stress response element [50], and the W-box for ABA and drought stress [51] were predicted in many of the GhPERK gene promoter regions. In our study, most of GhPERK genes have features typical of genes involved in growth and the responses to various stresses. For example, AtPERK4 is involved in ABA-responsive root-tip growth in Arabidopsis [16,17]. Previously, gene families in cotton and other plants were found to have similar cis-elements in their promoters and were shown to be functionally relevant $[52,53]$.

In our results, the uneven distribution of GhPERK genes on the At and Dt chromosomes of G. hirsutum indicates possible gene loss or addition through segmental or whole genome duplication events and incomplete genome sequencing could be an issue here as well. In addition, all GhPERK genes have no introns and the proteins have similar patterns of protein motif distribution. Previous studies have demonstrated the importance of introns in the evolution of various plant genes [33]. Many gene families indicate less or lack of introns in their gene families [34,52,54]. These exon/intron structural differences might be due to insertion/deletion events and are helpful in the identification of evolutionary mechanisms [55]. Introns are considered to be under weak selection pressure and the absence of introns indicates that GhPERK genes might have evolved relatively rapidly. Furthermore, the number of introns present early in gene family evolution were reduced over time, resulting in gene families with fewer or no introns [56] and these families are considered to be evolutionarily advanced [35], while gene families with more or larger introns are thought to have gained new functions during evolution.

It has been established that tandem duplication might cause an increase in intron numbers and create new genes [57]. Consistent with our findings, we did not identify and tandem duplication event in this study. Exon/intron structural conservation is functionally important and despite low levels of sequence conservation, the exon/intron structures in many gene families are conserved [58]. We speculate that the GhPERK gene family is an advanced gene family in which the members lost their introns over time but underwent expansion in a rapid phase of evolution.

\subsection{GhPERK Gene Family Duplication and Expansion}

G. hirsutum is an allotetraploid cotton species that is cultivated globally and accounts for $95 \%$ of global production [59]. The A- and D-genome cottons (G. arboreum and G. raimondii, respectively) experienced whole genome duplication and, as the result of hybridization, the allotetraploid AtDt genome species G. hirsutum emerged between 5 and 10 mya (million years ago) [41]. As allotetraploid 
crop, G. hirsutum is one of the best model species in which to study polyploidy in plants [28]. Gene duplication and divergence mainly lead toward evolutionary momentum [36]. Gene duplication generates functional divergence, which is essential for environmental adaptability and speciation [37]. Duplication is indicated when aligned sequences share more than $70 \%$ identity and cover more than $80 \%$ of the total length [38]. Two duplicated genes positioned on the same chromosome might be the result of tandem duplication, while their presence on different chromosomes of the same sub-genome is referred to as segmental duplication [60]. In segmental duplications, the duplicated genes are usually scattered throughout the genome, while tandemly duplicated genes tend to cluster together [61]. Approximately 65 mya, segmental along with whole genome duplications in ancestral plants contributed to the expansion of many gene families [62,63]. During evolution, two large scale segmental as well as small scale tandem duplications generated new genes that contributed genomic complexity to the plant kingdom [64].

In the present we study identified 33 GhPERK genes, double the number of PERK genes found in any other plant species, which demonstrates the effect of polyploidy on the PERK gene family in G. hirsutum. Polyploidy is a feature of genome evolution in G. hirsutum that consequently doubled the size of the PERK family as a result of segmental and whole genome duplication (WGD). As previously reported, polyploidy enabled many plant species to adapt to new environments [65]. The dramatic increase in the size of the GhPERK gene family can be evaluated by examining the PERK genes in the A- (G. arboreum) and D-genome (G. raimondii) cottons. Gene loss is an essential process that occurs after the hybridization event during genomic arrangements and chromosome doubling [26]. Even though all the other species included in our study also have many duplicated gene pairs, our phylogenetic analysis indicates that expansion of the PERK gene family in plants could be the result of segmental and whole genome duplication. Moreover, the G. hirsutum genome experienced fewer arrangements compared to paleopolyploid maize and Brassica [66,67].

Generally, polyploidy is associated with gene duplication and in our study we found that segmental and whole genome duplication were the main factors responsible for doubling the number of GhPERK genes. Segmental duplication is the main impetus during evolution and it has occurred in many plant genomes that contain many duplicated chromosomal blocks [64]. For example, many Arabidopsis gene families experienced coherent evolutionary dynamics that led to gene family expansion $[68,69]$. Furthermore, many gene families such as cotton WOX, YABBY, GRAS, RH2FE3, MIKC-Type and MADS-Box, soybean WRKY and sesame heat shock proteins experienced expansion as the result of segmental and whole genome duplication [31,45,52,54,70-72]. Our results showed that many of the GhPERK genes clustered into pairs, which indicates an ancient genome duplication event. The two copies of a gene can be subjected to shuffling and rearrangements that create potential diversity. Four possible fates have been described for duplicated genes [73]. One, one gene copy might be deleted during the evolutionary process, thereby removing functional redundancy. Two, sub-functionalization of both genes that shared the parental functions, leading to the development of partially different functions over time. Three, neo-functionalization in which one gene copy acquired new functions during evolution. Four, an intermediate form of sub- and neo-functionalization in which only genes critical for plant growth and development are retained. Thus, the large numbers of PERK genes in the G. hirsutum genome may be important for the plant's normal growth and development and in responding to phytohormone and abiotic stresses. In our study, we found that the GhPERK genes experienced strong purifying selection.

Arabidopsis experienced whole genome duplication twice in the Brassicaceae lineage [74]. In Addition, cotton and cacao share a common ancestor that experienced an ancient duplication 18-58 mya, eventually nascent duplication event in cotton again [25]. Orthologous/paralogous and gene duplication analysis showed that A-genome and At sub-genome PERK genes share a common ancestor, while D-genome and Dt sub-genome PERK genes belong to the same ancestor. Our investigations into cotton PERK gene family expansion provides useful information on chromosomal interaction and polyploidization in addition to differential rates of evolution 
and information transfer through inter-genomic hereditary. Taking all of our data together, we conclude that the cotton PERK gene family experienced segmental and whole genome duplication, which subsequently caused expansion of the GhPERK gene family.

\subsection{GhPERK Genes are Regulated by Abiotic and Hormonal Stresses}

A limited number of studies have been conducted to investigate the molecular and physiological functions of PERK proteins in plants. Previously, it has been reported that receptor-like protein kinases (RLKs) are likely to respond to external challenges in an-ever changing environment and are involved in hormonal response pathways, plant growth and development, self-incompatibility, cell differentiation, symbiosis and pathogen recognition [1]. In Arabidopsis, PERK1 was shown to be induced rapidly in response to wounding [14]. PERK4 a novel regulator of $\mathrm{Ca}^{2+}$ signaling, mediates the early events of the abscisic acid response in root tip growth $[16,17]$. However, no systematic study of PERK genes in cotton has been published at present.

The results of our study show that all predicted GhPERK genes are highly expressed in leaves and some stages of ovule development, demonstrating the positive roles that these genes may play in cotton leaf morphology with no obvious contribution to fiber development, implying a negative role in fiber development. Also, the expression of GhPERK genes can be significantly regulated (positively or negatively) by abiotic stress treatments such as cold, heat, salt, and PEG because all of the GhPERK genes displayed ubiquitous expression patterns that did not follow any particular fashion. Our qRT-PCR results agreed with previously published RNA-seq and transcriptomic data. Coupled with these findings, we conclude that GhPERK genes may provide important genetic strategies for breeding cotton lines that are tolerant to several abiotic stresses.

Further experiments indicated that the GhPERK genes could play a role in negative regulation of the brassinosteroid signaling pathway; the expression of all genes was up-regulated in all examined tissues in the cotton brassinosteroid mutant Pag1 compared to WT plants. Moreover, the expression of all tested GhPERK genes could be either up- or down-regulated by the active form of brassinosteroid, BL and by treatment with the two brassinosteroid biosynthesis inhibitors BRZ and PCZ. These findings show that further investigations are needed to explore the functions of GhPERK genes in the brassinosteroid signaling pathway. Additionally, all GhPERK genes showed significant responses upon exposure to several phytohormones including GA, IAA, SA and MeJA; the expression of GhPERK8, GhPERK9, GhPERK12, GhPERK23, GhPERK27 and GhPERK29 was up-regulated with a few exceptions, while GhPERK5 was down-regulated when the plants were exposed to all four plant hormones at all time points from 0.5 to 5 hours, supporting our hypothesis that GhPERK genes play essential roles in certain hormone signaling pathways. We can also conclude that the GhPERK genes experienced strong functional divergence following gene duplication during the evolution of the GhPERK gene family in cotton.

\subsection{GhPERK co-expressed Genes Showed Functional Divergance}

Proline-rich extensin-like receptor kinases (PERKs) are an important class of receptor kinases. These are associated with multiple molecular and cellular mechanisms affecting developmental, signaling and physiological processes in plants [15]. In the current study, a co-expressed network indicated that two PERK genes were co-expressed with one or more other G. hirsutum genes. Gene Ontology (GO) showed that these genes were related to enzyme inhibitor activity (GO:0004857), polygalacturonase activity (GO:0004650), glycoprotein binding (GO:0005515), calcium ion binding (GO:0005509), actin binding (GO:0003779), zinc ion binding (GO:0008270) and defense response (GO:0006952). Additionally, a KEGG enrichment analysis revealed that these two PERK genes could be involved in plant-pathogen interaction (K02183), pentose and glucuronate interconversions (K01051), regulation of actin cytoskeleton (K05765), nitrogen metabolism (K01674), monoterpenoid biosynthesis (K07385) and microbial metabolism in diverse environments (K00860). These results indicated that 
PERK genes could be involved in several molecular, biological and physiological processes that might be the result of $P E R K$ genes functional divergence during long evolutionary history of cotton.

\section{Materials and Methods}

\subsection{Identification of PERK Protein Family Members}

The PERK genes and encoded proteins in other species were identified via a BLAST search using the Arabidopsis PERK genes [15] and encoded protein sequences as queries in G. arboreum (ICR, version 1.0), G. hirsutum (NAU, version 1.1), G. raimondii (JGI, version 2.0), G. max (version 10), P. trichocarpa (version 2.0), T. cacao (version 10), O. sativa (version 10), Z. mays (version 10), S. bicolor (version 10), A. comosus (version 3.0), C. reinhardtii (version 5.5), P. patens (version 3.3), S. moellendorffii (version 1.0) and P. taeda (version 1.0). Moreover, the definition of the PERK (PF07714) domain was downloaded from Pfam: http:/ /pfam.janelia.org/ [75] and then the hidden Markov model (HMM) was used to verify the PERKs from other species. The G. arboreum database was downloaded from (ftp://bioinfo. ayit.edu.cn/downloads /) while G. raimondii and G. hirsutum were downloaded from COTTONGEN (https://www.cottongen.org/). Also, Arabidopsis database was downloaded from TAIR 10 (http: / / www.Arabidopsis.org), while sequence databases for the other plant species were downloaded from online Phytozome v11 (https:/ / phytozome.jgi.doe.gov/pz/portal.html). All retrieved sequences were verified by SMART (http:/ / smart.embl-heidelberg.de/) [76] and Interproscan 63.0 (http:/ / www.ebi. ac.uk/InterProScan/) [77]. The biophysical properties were predicted with the ExPASy ProtParam tool (http:/ / us.expasy.org/tools/protparam.html) and subcellular localization was predicted using softberry (www.softberry.com).

\subsection{Sequence Logos and Phylogenetic Analysis}

Sequence logos for conserved amino acid residues and phylogenetic analysis were performed as described previously [45]. In brief, the sequence logos were constructed using online tool WEBLOG [39], while the phylogenetic tree was generated with MEGA 7.0 [78] using the Maximum likelihood (ML) method. Bootstrapping with 1000 replicates was used to test the statistical support of the nodes in the phylogenetic trees. The default parameters were used to generate the sequence logos and phylogenetic trees used in this study.

\subsection{Exon/Intron, Protein Motifs and Promoter cis-elements Analysis}

To determine exon/intron structure, bed-files obtained from databases were analyzed using GSDS 2.0 (http:/ / gsds.cbi.pku.edu.cn/index.php) [79] and the NJ tree was constructed with MEGA 7.0 as described above. Protein motif distribution patterns were determined using the online MEME tool (http:/ / meme-suite.org/tools/meme) [80] as described [81]. For cis-element analysis in promoter regions, 2-kb upstream sequences were analyzed in PlantCARE [82] and the elements were categorized based on their predicted functions.

\subsection{Genomic Distribution, Gene Duplication and Synteny Analysis}

To determine the chromosomal distribution of GhPERK genes, extracted gff3-files of cotton genome annotation data (ftp:/ / ftp.bioinfo.wsu.edu/species/Gossypium_hirsutum/NAU-NBI_G) were used to map the genes to the chromosomes using MapInspect (http:/ / www.plantbreeding.wur. $\mathrm{nl} / \mathrm{UK} /$ software_mapinspect.html) [83]. Gene duplication analysis and $\mathrm{Ka} / \mathrm{Ks}$ values were calculated using methods described previously [45]. In short, CIRCOS [84] was used to generate the figure and $\mathrm{Ka} / \mathrm{Ks}$ values were calculated using PAL2NAL (http:/ /www.bork.embl.de/pal2nal/) [85] and the CODEML program in the PAML package [86]. 


\subsection{Expression Analysis, Stress Treatments, $q R T-P C R$ and Co-expression Network Analysis}

In our study, the G. hirsutum variety CRI24 and the cotton brassinosteroid mutant Pag1 were used for gene expression analysis. To determine the tissue-specific expression patterns, all sampled tissues were obtained from cotton plants grown under field conditions with standard cultural practices. Abiotic stress treatments were conducted as described previously [31,54]. For hormonal stresses, the seedlings were treated with BL $(10 \mu \mathrm{M})$, BRZ $(10 \mu \mathrm{M})$, PCZ $(10 \mu \mathrm{M})$, GA $(100 \mu \mathrm{M})$, IAA $(100 \mu \mathrm{M})$, SA $(10 \mu \mathrm{M})$ and MeJA $(10 \mu \mathrm{M})$ for $0.5,1,3$ and $5 \mathrm{~h}$. Seedlings collected at 0 hour were used as the control (CK). All samples were immediately frozen in liquid nitrogen and kept at $-80^{\circ} \mathrm{C}$ prior to use.

Total RNA was extracted from cotton samples using the RNAprep Pure Plant Kit (Tiangen, Beijing, China) and cDNA was synthesized from $1 \mu \mathrm{g}$ total RNA using the Prime-Script ${ }^{\circledR R T}$ reagent kit (Takara, Dalian, China). The GhHis3 gene (GenBank accession no. AF024716) was used as the internal control for normalization of gene expression and the qRT-PCR assays were performed with SYBR Green on a LightCycler 480 system (Roche Diagnostics GmbH, Sandhofer Straße 116, 68305 Mannheim, Germany). All the primers used in this study are given in Table S7. The relative expression values were calculated using the method described previously [87]. Co-expression data for GhPERK genes was downloaded from online tool ccNET [88] (http:/ / structuralbiology.cau.edu.cn/gossypium/cytoscape/network.list. php) and finally, we visualized co-expression network using Cytoscape (version 3.4.0) program [89]. Additionally, the putative functions of co-expressed genes based on Gene Ontology (GO) and Kyoto Encyclopedia of Genes and Genomes (KEGG) enrichment analyses were investigated from online tool CottonFGD (http:/ /www.cottonfgd.org/analyze/).

\section{Conclusions}

The present study identified 207 PERK gene family members in 15 different plant species. Among these genes, we identified 33 GhPERK genes from the allotetraploid cotton species G. hirsutum. Sequence analysis of conserved amino acid residues among the PERK proteins from Arabidopsis, rice and G. hirsutum showed that PERK genes are highly conserved in plants. Phylogenetic analysis grouped all PERK proteins into four clades on the basis of sequence homology. GhPERK genes do not have introns and the protein motif distribution pattern is conserved across all proteins. Furthermore, the predicted cis-elements in the GhPERK gene promoter regions indicate their functional relevance to growth, development and the response to various stresses. Uneven distribution of the GhPERK genes on the At and Dt chromosomes of G. hirsutum may be due to gene loss or addition through segmental or whole genome duplication during evolution, and/or incomplete genome sequencing. Also, gene duplication and synteny analysis indicates that the cotton PERK genes experienced segmental and whole genome duplication during evolution that subsequently resulted in a major expansion of the GhPERK gene family. Additionally, our data shows that the GhPERK genes experienced functional divergence during evolution and their high levels of expression in leaves can be regulated by abiotic and hormonal stresses, indicating that members of this gene family could play crucial roles in hormone signaling pathways, especially for the brassinosteroids. Moreover, PERK genes could be involved in several molecular, biological and physiological processes that might be the result of PERK genes functional divergence during long evolutionary history of cotton.

Supplementary Materials: The following are available online at http:/ /www.mdpi.com/1422-0067/20/7/1750/ s1. Figure S1. Phylogenetic analysis and tree was generated by NJ method among cotton PERK genes including G. arboreum, G. hirsutum and G. raimondii in order to estimate common ancestor hypothesis. Tree divided all PERK genes into six groups represented by different colors. Figure S2. Exon/intron and protein motif distribution of GhPERK genes couples with phylogenetic tree constructed by NJ method. (A) Exon/intron distribution of GhPERK genes. (B) Protein motif distribution of GhPERK genes. Figure S3. Chromosomal distribution of GhPERK genes on their corresponding chromosomes. A01 to A13 and D01 to D13 represents At and Dt sub-genomes G. hirsutum chromosomes, respectively. Chromosomal sizes were calculated from published genome data. Table S1. Gene locus ID and their proposed names of all observed species including A. thaliana, G. arboreum, G. hirsutum, G. max, G. raimondii, O. sativa, P. trichocarpa, S. bicolor, , T. cacao and Z. mays. Table S2. Biophysical properties of GhPERK genes including locus ID, start and end point, strand, CDs (coding sequence), protein length, MW (molecular weight), pl (isoelectric point), gravity and predicted subcellular localization. Table S3. 
Promoter cis-element prediction of GhPERK genes was characterized according to their relatedness to growth and development, light and different stresses. Table S4. Orthologous/paralogous in At, Dt sub-genomes of G. hirsutum, G. arboreum (A genome) and G. raimondii (D genome). Ka/Ks (non-synonymous/synonymous) values of all identified orthologous/paralogous gene pairs were also mentioned. Table S5. RNA-seq data analysis of GhPERK genes in fuzzless/lintless mutants (M1l and M2l). Additionally, genes were classified depending on their up or down regulated expression pattern. Table S6. Gene Ontology (GO) and Kyoto Encyclopedia of Genes and Genomes (KEGG) enrichment analysis of other G. hirsutum co-expressed genes with GhPERKs. Table S7. List of all oligonucleotide primers used in this study.

Author Contributions: Z.Y. and Z.W.: conceived and designed the experiments; G.Q., J.L., D.Y., Z.L., L.L., H.M. and S.M.: performed the experiments and analyzed the data; G.Q., L.J., Z.Y. and Z.W.: drafted the manuscript; L.L., H.M. and S.M.: revised the manuscript. All authors have read and approved the final manuscript.

Funding: This work was supported by the Major Research Plan of National Natural Science Foundation of China (NO.31690093).

Acknowledgments: We would like to thank the reviewers for their comments and suggestion.

Conflicts of Interest: The authors declare that they have no competing interests.

\section{Abbreviations}

$\begin{array}{ll}\text { NaCl } & \text { Sodium chloride } \\ \text { PEG } & \text { Polyethylene glycol } \\ \text { GA } & \text { Gibberellic acid } \\ \text { IAA } & \text { Indole-3-acetic acid } \\ \text { BL } & \text { Brassinolide } \\ \text { BRZ } & \text { Brassinazole } \\ \text { PCZ } & \text { Propiconazole } \\ \text { MeJA } & \text { Methyl jasmonate } \\ \text { SA } & \text { Salicylic acid } \\ \text { DPA } & \text { Days post-anthesis } \\ \text { MM } & \text { Micro-molar } \\ \text { Mya } & \text { Million years ago } \\ \text { G. arboreum } & \text { Gossypium arboreum } \\ \text { G. hirsutum } & \text { Gossypium hirsutum } \\ \text { G. raimondii } & \text { Gossypium raimondii } \\ \text { Z. mays } & \text { Zea mays } \\ \text { O. sativa } & \text { Oryza sativa } \\ \text { G. max } & \text { Glycine max } \\ \text { A. thaliana } & \text { Arabidopsis thaliana } \\ \text { P. trichocarpa } & \text { Populus trichocarpa } \\ \text { T. cacao } & \text { Theobroma cacao } \\ \text { S. bicolor } & \text { Sorghum bicolor }\end{array}$

\section{References}

1. Morris, E.R.; Walker, J.C. Receptor-like protein kinases: The keys to response. Curr. Opin. Plant Biol. 2003, 6, 339-342. [CrossRef]

2. Shiu, S.H.; Bleecker, A.B. Receptor-like kinases from arabidopsis form a monophyletic gene family related to animal receptor kinases. Proc. Natl. Acad. Sci. USA 2001, 98, 10763-10768. [CrossRef] [PubMed]

3. Shiu, S.H.; Karlowski, W.M.; Pan, R.; Tzeng, Y.H.; Mayer, K.F.; Li, W.H. Comparative analysis of the receptor-like kinase family in arabidopsis and rice. Plant Cell 2004, 16, 1220-1234. [CrossRef] [PubMed]

4. Dievart, A.; Clark, S.E. Lrr-containing receptors regulating plant development and defense. Development 2004, 131, 251-261. [CrossRef]

5. Shiu, S.H.; Bleecker, A.B. Plant receptor-like kinase gene family: Diversity, function and signaling. Sci. STKE 2001, 2001, re22. [CrossRef] [PubMed]

6. Shiu, S.H.; Bleecker, A.B. Expansion of the receptor-like kinase/pelle gene family and receptor-like proteins in arabidopsis. Plant Physiol. 2003, 132, 530-543. 
7. Li, J.; Chory, J. A putative leucine-rich repeat receptor kinase involved in brassinosteroid signal transduction. Cell 1997, 90, 929-938. [CrossRef]

8. Li, J.; Wen, J.; Lease, K.A.; Doke, J.T.; Tax, F.E.; Walker, J.C. Bak1, an arabidopsis lrr receptor-like protein kinase, interacts with bri1 and modulates brassinosteroid signaling. Cell 2002, 110, 213-222. [CrossRef]

9. Nam, K.H.; Li, J. Bri1/bak1, a receptor kinase pair mediating brassinosteroid signaling. Cell 2002, 110, 203-212. [CrossRef]

10. Champion, A.; Kreis, M.; Mockaitis, K.; Picaud, A.; Henry, Y. Arabidopsis kinome: After the casting. Funct. Integr. Genom. 2004, 4, 163-187. [CrossRef] [PubMed]

11. Shpak, E.D.; Berthiaume, C.T.; Hill, E.J.; Torii, K.U. Synergistic interaction of three erecta-family receptor-like kinases controls arabidopsis organ growth and flower development by promoting cell proliferation. Development 2004, 131, 1491-1501. [CrossRef] [PubMed]

12. Dievart, A.; Dalal, M.; Tax, F.E.; Lacey, A.D.; Huttly, A.; Li, J.; Clark, S.E. CLAVATA1 dominant-negative alleles reveal functional overlap between multiple receptor kinases that regulate meristem and organ development. Plant Cell 2003, 15, 1198-1211. [CrossRef]

13. Shpak, E.D.; Lakeman, M.B.; Torii, K.U. Dominant-negative receptor uncovers redundancy in the arabidopsis erecta leucine-rich repeat receptor-like kinase signaling pathway that regulates organ shape. Plant Cell 2003, 15, 1095-1110. [CrossRef] [PubMed]

14. Silva, N.F.; Goring, D.R. The proline-rich, extensin-like receptor kinase-1 (perk1) gene is rapidly induced by wounding. Plant Mol. Biol. 2002, 50, 667-685. [CrossRef] [PubMed]

15. Nakhamchik, A.; Zhao, Z.; Provart, N.J.; Shiu, S.H.; Keatley, S.K.; Cameron, R.K.; Goring, D.R. A comprehensive expression analysis of the arabidopsis proline-rich extensin-like receptor kinase gene family using bioinformatic and experimental approaches. Plant Cell Physiol. 2004, 45, 1875-1881. [CrossRef]

16. Bai, L.; Zhang, G.; Zhou, Y.; Zhang, Z.; Wang, W.; Du, Y.; Wu, Z.; Song, C.P. Plasma membrane-associated proline-rich extensin-like receptor kinase 4 , a novel regulator of ca signalling, is required for abscisic acid responses in arabidopsis thaliana. Plant J. 2009, 60, 314-327. [CrossRef]

17. Bai, L.; Zhou, Y.; Song, C.P. Arabidopsis proline-rich extensin-like receptor kinase 4 modulates the early event toward abscisic acid response in root tip growth. Plant Signal. Behav. 2009, 4, 1075-1077. [CrossRef]

18. Kim, H.J.; Triplett, B.A. Cotton fiber germin-like protein. I. Molecular cloning and gene expression. Planta 2004, 218, 516-524.

19. Sun, Y.; Veerabomma, S.; Abdel-Mageed, H.A.; Fokar, M.; Asami, T.; Yoshida, S.; Allen, R.D. Brassinosteroid regulates fiber development on cultured cotton ovules. Plant Cell Physiol. 2005, 46, 1384-1391. [CrossRef] [PubMed]

20. Yang, Z.R.; Zhang, C.J.; Yang, X.J.; Liu, K.; Wu, Z.X.; Zhang, X.Y.; Zheng, W.; Xun, Q.Q.; Liu, C.L.; Lu, L.L.; et al. Pag1, a cotton brassinosteroid catabolism gene, modulates fiber elongation. New Phytol. 2014, 203, 437-448. [CrossRef] [PubMed]

21. Du, X.M.; Huang, G.; He, S.P.; Yang, Z.E.; Sun, G.F.; Ma, X.F.; Li, N.; Zhang, X.Y.; Sun, J.L.; Liu, M.; et al. Resequencing of 243 diploid cotton accessions based on an updated a genome identifies the genetic basis of key agronomic traits. Nat. Genet. 2018, 50, 796-802. [CrossRef]

22. Ma, Z.; He, S.; Wang, X.; Sun, J.; Zhang, Y.; Zhang, G.; Wu, L.; Li, Z.; Liu, Z.; Sun, G.; et al. Resequencing a core collection of upland cotton identifies genomic variation and loci influencing fiber quality and yield. Nat. Genet. 2018, 50, 803-813. [CrossRef]

23. Wang, M.; Wang, P.; Lin, M.; Ye, Z.; Li, G.; Tu, L.; Shen, C.; Li, J.; Yang, Q.; Zhang, X. Evolutionary dynamics of 3d genome architecture following polyploidization in cotton. Nat. Plants 2018, 4, 90-97. [CrossRef]

24. Zhang, T.Z.; Hu, Y.; Jiang, W.K.; Fang, L.; Guan, X.Y.; Chen, J.D.; Zhang, J.B.; Saski, C.A.; Scheffler, B.E.; Stelly, D.M.; et al. Sequencing of allotetraploid cotton (Gossypium hirsutum L. acc. TM-1) provides a resource for fiber improvement. Nat. Biotechnol. 2015, 33, 531-537. [CrossRef]

25. Li, F.G.; Fan, G.Y.; Wang, K.B.; Sun, F.M.; Yuan, Y.L.; Song, G.L.; Li, Q.; Ma, Z.Y.; Lu, C.R.; Zou, C.S.; et al. Genome sequence of the cultivated cotton gossypium arboreum. Nat. Genet. 2014, 46, 567-572. [CrossRef]

26. Paterson, A.H.; Wendel, J.F.; Gundlach, H.; Guo, H.; Jenkins, J.; Jin, D.C.; Llewellyn, D.; Showmaker, K.C.; Shu, S.Q.; Udall, J.; et al. Repeated polyploidization of gossypium genomes and the evolution of spinnable cotton fibres. Nature 2012, 492, 423-427. [CrossRef]

27. Higo, K.; Ugawa, Y.; Iwamoto, M.; Korenaga, T. Plant cis-acting regulatory DNA elements (place) database: 1999. Nucleic Acids Res. 1999, 27, 297-300. [CrossRef] 
28. Wendel, J.F.; Cronn, R.C. Polyploidy and the evolutionary history of cotton. Adv. Agron. 2003, 78, $139-186$.

29. Ruprecht, C.; Proost, S.; Hernandez-Coronado, M.; Ortiz-Ramirez, C.; Lang, D.; Rensing, S.A.; Becker, J.D.; Vandepoele, K.; Mutwil, M. Phylogenomic analysis of gene co-expression networks reveals the evolution of functional modules. Plant J. 2017, 90, 447-465. [CrossRef]

30. Bao, Y.; Hu, G.J.; Flagel, L.E.; Salmon, A.; Bezanilla, M.; Paterson, A.H.; Wang, Z.N.; Wendel, J.F. Parallel up-regulation of the profilin gene family following independent domestication of diploid and allopolyploid cotton (Gossypium). Proc. Natl. Acad. Sci. USA 2011, 108, 21152-21157. [CrossRef]

31. Yang, Z.E.; Gong, Q.; Wang, L.L.; Jin, Y.Y.; Xi, J.P.; Li, Z.; Qin, W.Q.; Yang, Z.R.; Lu, L.L.; Chen, Q.J.; et al. Genome-wide study of yabby genes in upland cotton and their expression patterns under different stresses. Front. Genet. 2018, 9, 33. [CrossRef]

32. Maestrini, P.; Cavallini, A.; Rizzo, M.; Giordani, T.; Bernardi, R.; Durante, M.; Natali, L. Isolation and expression analysis of low temperature-induced genes in white poplar (Populus alba). J. Plant Physiol. 2009, 166, 1544-1556. [CrossRef]

33. Roy, S.W.; Gilbert, W. The evolution of spliceosomal introns: Patterns, puzzles and progress. Nat. Rev. Genet. 2006, 7, 211-221.

34. Serrano, M.; Parra, S.; Alcaraz, L.D.; Guzman, P. The ATL gene family from Arabidopsis thaliana and Oryza sativa comprises a large number of putative ubiquitin ligases of the ring-h2 type. J. Mol. Evol. 2006, 62, 434-445. [CrossRef]

35. Roy, S.W.; Gilbert, W. Complex early genes. Proc. Natl. Acad. Sci. USA 2005, 102, 1986-1991. [CrossRef]

36. Chothia, C.; Gough, J.; Vogel, C.; Teichmann, S.A. Evolution of the protein repertoire. Science 2003, 300, 1701-1703. [CrossRef]

37. Conant, G.C.; Wolfe, K.H. Turning a hobby into a job: How duplicated genes find new functions. Nat. Rev. Genet. 2008, 9, 938-950. [CrossRef]

38. Yang, S.H.; Zhang, X.H.; Yue, J.X.; Tian, D.C.; Chen, J.Q. Recent duplications dominate nbs-encoding gene expansion in two woody species. Mol. Genet. Genom. 2008, 280, 187-198. [CrossRef]

39. Crooks, G.E.; Hon, G.; Chandonia, J.M.; Brenner, S.E. Weblogo: A sequence logo generator. Genome Res. 2004, 14, 1188-1190. [CrossRef]

40. Higo, K.; Ugawa, Y.; Iwamoto, M.; Higo, H. PLACE: A database of plant cis-acting regulatory DNA elements. Nucleic Acids Res. 1998, 26, 358-359. [CrossRef]

41. Li, F.; Fan, G.; Lu, C.; Xiao, G.; Zou, C.; Kohel, R.J.; Ma, Z.; Shang, H.; Ma, X.; Wu, J.; et al. Genome sequence of cultivated upland cotton (Gossypium hirsutum TM-1) provides insights into genome evolution. Nat. Biotechnol. 2015, 33, 524-530. [CrossRef]

42. Prince, V.E.; Pickett, F.B. Splitting pairs: The diverging fates of duplicated genes. Nat. Rev. Genet. 2002, 3, 827-837. [CrossRef]

43. Naoumkina, M.; Thyssen, G.N.; Fang, D.D. Rna-seq analysis of short fiber mutants ligon-lintless-1 (li 1) and -2 (li 2) revealed important role of aquaporins in cotton (Gossypium hirsutum L.) fiber elongation. BMC Plant Biol. 2015, 15, 65. [CrossRef]

44. Aoki, K.; Ogata, Y.; Shibata, D. Approaches for extracting practical information from gene co-expression networks in plant biology. Plant Cell Physiol. 2007, 48, 381-390. [CrossRef]

45. Yang, Z.E.; Gong, Q.; Qin, W.Q.; Yang, Z.R.; Cheng, Y.; Lu, L.L.; Ge, X.Y.; Zhang, C.J.; Wu, Z.X.; Li, F.G. Genome-wide analysis of wox genes in upland cotton and their expression pattern under different stresses. Bmc Plant Biol. 2017, 17, 113. [CrossRef]

46. Fankhauser, C.; Chory, J. Light control of plant development. Annu. Rev. Cell Dev. Biol. 1997, 13, $203-229$. [CrossRef]

47. Wen, F.; Zhu, H.; Li, P.; Jiang, M.; Mao, W.Q.; Ong, C.; Chu, Z.Q. Genome-wide evolutionary characterization and expression analyses of wrky family genes in brachypodium distachyon. DNA Res. 2014, 21, 327-339. [CrossRef]

48. Song, C.P.; Agarwal, M.; Ohta, M.; Guo, Y.; Halfter, U.; Wang, P.C.; Zhu, J.K. Role of an arabidopsis ap2/erebp-type transcriptional repressor in abscisic acid and drought stress responses. Plant Cell 2005, 17, 2384-2396. [CrossRef]

49. Narusaka, Y.; Nakashima, K.; Shinwari, Z.K.; Sakuma, Y.; Furihata, T.; Abe, H.; Narusaka, M.; Shinozaki, K.; Yamaguchi-Shinozaki, K. Interaction between two cis-acting elements, abre and dre, in aba-dependent expression of Arabidopsis $r d 29 A$ gene in response to dehydration and high-salinity stresses. Plant J. 2003, 34, 137-148. [CrossRef] 
50. Diaz-Martin, J.; Almoguera, C.N.; Prieto-Dapena, P.; Espinosa, J.M.; Jordano, J. Functional interaction between two transcription factors involved in the developmental regulation of a small heat stress protein gene promoter. Plant Physiol. 2005, 139, 1483-1494. [CrossRef]

51. Singh, K.B.; Foley, R.C.; Onate-Sanchez, L. Transcription factors in plant defense and stress responses. Curr. Opin. Plant Biol. 2002, 5, 430-436. [CrossRef]

52. Qanmber, G.; Yu, D.; Li, J.; Wang, L.; Ma, S.; Lu, L.; Yang, Z.; Li, F. Genome-wide identification and expression analysis of gossypium ring-h2 finger e3 ligase genes revealed their roles in fiber development and phytohormone and abiotic stress responses. J. Cotton Res. 2018, 1, 1. [CrossRef]

53. Pandey, A.; Misra, P.; Alok, A.; Kaur, N.; Sharma, S.; Lakhwani, D.; Asif, M.H.; Tiwari, S.; Trivedi, P.K. Genome-wide identification and expression analysis of homeodomain leucine zipper subfamily iv (hdz iv) gene family from musa accuminata. Front. Plant Sci. 2016, 7, 20. [CrossRef]

54. Zhang, B.; Liu, J.; Yang, Z.E.; Chen, E.Y.; Zhang, C.J.; Zhang, X.Y.; Li, F.G. Genome-wide analysis of gras transcription factor gene family in Gossypium hirsutum L. BMC Genom. 2018, 19, 348. [CrossRef]

55. Lecharny, A.; Boudet, N.; Gy, I.; Aubourg, S.; Kreis, M. Introns in, introns out in plant gene families: A genomic approach of the dynamics of gene structure. J. Struct. Funct. Genom. 2003, 3, 111-116. [CrossRef]

56. Roy, S.W.; Penny, D. A very high fraction of unique intron positions in the intron-rich diatom thalassiosira pseudonana indicates widespread intron gain. Mol. Biol. Evol. 2007, 24, 1447-1457. [CrossRef]

57. Iwamoto, M.; Maekawa, M.; Saito, A.; Higo, H.; Higo, K. Evolutionary relationship of plant catalase genes inferred from exon-intron structures: Isozyme divergence after the separation of monocots and dicots. Theor. Appl. Genet. 1998, 97, 9-19. [CrossRef]

58. Frugoli, J.A.; McPeek, M.A.; Thomas, T.L.; McClung, C.R. Intron loss and gain during evolution of the catalase gene family in angiosperms. Genetics 1998, 149, 355-365.

59. Tiwari, S.C.; Wilkins, T.A. Cotton (Gossypium hirsutum) seed trichomes expand via diffuse growing mechanism. Can. J. Bot.-Rev. Can. De Bot. 1995, 73, 746-757. [CrossRef]

60. He, H.S.; Dong, Q.; Shao, Y.H.; Jiang, H.Y.; Zhu, S.W.; Cheng, B.J.; Xiang, Y. Genome-wide survey and characterization of the WRKY gene family in Populus trichocarpa. Plant Cell Rep. 2012, 31, 1199-1217. [CrossRef]

61. Schauser, L.; Wieloch, W.; Stougaard, J. Evolution of nin-like proteins in arabidopsis, rice and lotus japonicus. J. Mol. Evol. 2005, 60, 229-237. [CrossRef]

62. Barakat, A.; Bagniewska-Zadworna, A.; Choi, A.; Plakkat, U.; DiLoreto, D.S.; Yellanki, P.; Carlson, J.E. The cinnamyl alcohol dehydrogenase gene family in populus: Phylogeny, organization and expression. BMC Plant Biol. 2009, 9, 26. [CrossRef]

63. Wang, Z.C.; Zhang, H.Z.; Yang, J.L.; Chen, Y.L.; Xu, X.M.; Mao, X.L.; Li, C.H. Phylogenetic, expression and bioinformatic analysis of the ABC1 gene family in Populus trichocarpa. Sci. World J. 2013, 2013, 785070.

64. Cannon, S.B.; Mitra, A.; Baumgarten, A.; Young, N.D.; May, G. The roles of segmental and tandem gene duplication in the evolution of large gene families in Arabidopsis thaliana. BMC Plant Biol. 2004, 4, 10. [CrossRef]

65. Ramsey, J.; Schemske, D.W. Pathways, mechanisms and rates of polyploid formation in flowering plants. Annu. Rev. Ecol. Syst. 1998, 29, 467-501. [CrossRef]

66. Gaeta, R.T.; Pires, J.C.; Iniguez-Luy, F.; Leon, E.; Osborn, T.C. Genomic changes in resynthesized Brassica napus and their effect on gene expression and phenotype. Plant Cell 2007, 19, 3403-3417. [CrossRef]

67. Woodhouse, M.R.; Schnable, J.C.; Pedersen, B.S.; Lyons, E.; Lisch, D.; Subramaniam, S.; Freeling, M. Following tetraploidy in maize, a short deletion mechanism removed genes preferentially from one of the two homologs. PLoS Biol. 2010, 8, e1000409. [CrossRef]

68. Baumberger, N.; Doesseger, B.; Guyot, R.; Diet, A.; Parsons, R.L.; Clark, M.A.; Simmons, M.P.; Bedinger, P.; Goff, S.A.; Ringli, C.; et al. Whole-genome comparison of leucine-rich repeat extensins in arabidopsis and rice. A conserved family of cell wall proteins form a vegetative and a reproductive clade. Plant Physiol. 2003, 131, 1313-1326. [CrossRef]

69. Wang, D.; Guo, Y.H.; Wu, C.G.; Yang, G.D.; Li, Y.Y.; Zheng, C.C. Genome-wide analysis of ccch zinc finger family in arabidopsis and rice. BMC Genom. 2008, 9, 44. [CrossRef]

70. Dossa, K.; Diouf, D.; Cisse, N. Genome-wide investigation of hsf genes in sesame reveals their segmental duplication expansion and their active role in drought stress response. Front. Plant Sci. 2016, 7, 1522. [CrossRef] 
71. Yin, G.J.; Xu, H.L.; Xiao, S.Y.; Qin, Y.J.; Li, Y.X.; Yan, Y.M.; Hu, Y.K. The large soybean (Glycine max) WRKY TF family expanded by segmental duplication events and subsequent divergent selection among subgroups. BMC Plant Biol. 2013, 13, 148. [CrossRef]

72. Ren, Z.; Yu, D.; Yang, Z.; Li, C.; Qanmber, G.; Li, Y.; Li, J.; Liu, Z.; Lu, L.; Wang, L.; et al. Genome-wide identification of the MIKC-type MADS-box gene family in Gossypium hirsutum L. Unravels their roles in flowering. Front Plant Sci 2017, 8, 384. [CrossRef]

73. Charon, C.; Bruggeman, Q.; Thareau, V.; Henry, Y. Gene duplication within the green lineage: The case of tel genes. J. Exp. Bot. 2012, 63, 5061-5077. [CrossRef]

74. Wang, X.W.; Wang, H.Z.; Wang, J.; Sun, R.F.; Wu, J.; Liu, S.Y.; Bai, Y.Q.; Mun, J.H.; Bancroft, I.; Cheng, F.; et al. The genome of the mesopolyploid crop species Brassica rapa. Nat. Genet. 2011, 43, 1035-1039. [CrossRef]

75. Finn, R.D.; Coggill, P.; Eberhardt, R.Y.; Eddy, S.R.; Mistry, J.; Mitchell, A.L.; Potter, S.C.; Punta, M.; Qureshi, M.; Sangrador-Vegas, A.; et al. The pfam protein families database: Towards a more sustainable future. Nucleic Acids Res. 2016, 44, D279-D285. [CrossRef]

76. Letunic, I.; Doerks, T.; Bork, P. Smart: Recent updates, new developments and status in 2015. Nucleic Acids Res. 2015, 43, D257-D260. [CrossRef]

77. Jones, P.; Binns, D.; Chang, H.Y.; Fraser, M.; Li, W.; McAnulla, C.; McWilliam, H.; Maslen, J.; Mitchell, A.; Nuka, G.; et al. Interproscan 5: Genome-scale protein function classification. Bioinformatics 2014, 30, 1236-1240. [CrossRef]

78. Kumar, S.; Stecher, G.; Tamura, K. Mega7: Molecular evolutionary genetics analysis version 7.0 for bigger datasets. Mol. Biol. Evol. 2016, 33, 1870-1874. [CrossRef]

79. Hu, B.; Jin, J.; Guo, A.Y.; Zhang, H.; Luo, J.; Gao, G. GSDS 2.0: An upgraded gene feature visualization server. Bioinformatics 2015, 31, 1296-1297. [CrossRef]

80. Bailey, T.L.; Williams, N.; Misleh, C.; Li, W.W. Meme: Discovering and analyzing DNA and protein sequence motifs. Nucleic Acids Res. 2006, 34, W369-W373. [CrossRef]

81. Li, J.; Yu, D.; Qanmber, G.; Lu, L.; Wang, L.; Zheng, L.; Liu, Z.; Wu, H.; Liu, X.; Chen, Q.; et al. GhKLCR1, a kinesin light chain-related gene, induces drought-stress sensitivity in Arabidopsis. Sci. China Life Sci. 2018, 62, 63-75. [CrossRef]

82. Lescot, M.; Dehais, P.; Thijs, G.; Marchal, K.; Moreau, Y.; Van de Peer, Y.; Rouze, P.; Rombauts, S. Plantcare, a database of plant cis-acting regulatory elements and a portal to tools for in silico analysis of promoter sequences. Nucleic Acids Res. 2002, 30, 325-327. [CrossRef]

83. Jia, J.T.; Zhao, P.C.; Cheng, L.Q.; Yuan, G.X.; Yang, W.G.; Liu, S.; Chen, S.Y.; Qi, D.M.; Liu, G.S.; Li, X.X. Mads-box family genes in sheepgrass and their involvement in abiotic stress responses. BMC Plant Biol. 2018, 18, 42. [CrossRef]

84. Krzywinski, M.; Schein, J.; Birol, I.; Connors, J.; Gascoyne, R.; Horsman, D.; Jones, S.J.; Marra, M.A. Circos: An information aesthetic for comparative genomics. Genome Res. 2009, 19, 1639-1645. [CrossRef]

85. Suyama, M.; Torrents, D.; Bork, P. Pal2nal: Robust conversion of protein sequence alignments into the corresponding codon alignments. Nucleic Acids Res. 2006, 34, W609-W612. [CrossRef]

86. Yang, Z.H. Paml 4: Phylogenetic analysis by maximum likelihood. Mol. Biol. Evol. 2007, 24, $1586-1591$. [CrossRef]

87. Livak, K.J.; Schmittgen, T.D. Analysis of relative gene expression data using real-time quantitative pcr and the 2(-delta delta $\mathrm{c}(\mathrm{t}))$ method. Methods 2001, 25, 402-408. [CrossRef]

88. You, Q.; Xu, W.; Zhang, K.; Zhang, L.; Yi, X.; Yao, D.; Wang, C.; Zhang, X.; Zhao, X.; Provart, N.J.; et al. ccNET: Database of co-expression networks with functional modules for diploid and polyploid Gossypium. Nucleic Acids Res. 2017, 45, D1090-D1099. [CrossRef]

89. Shannon, P.; Markiel, A.; Ozier, O.; Baliga, N.S.; Wang, J.T.; Ramage, D.; Amin, N.; Schwikowski, B.; Ideker, T. Cytoscape: A software environment for integrated models of biomolecular interaction networks. Genome Res. 2003, 13, 2498-2504. [CrossRef]

(c) 2019 by the authors. Licensee MDPI, Basel, Switzerland. This article is an open access article distributed under the terms and conditions of the Creative Commons Attribution (CC BY) license (http:/ / creativecommons.org/licenses/by/4.0/). 